Journal of

Entreprenuership and

Project Management

(JEPM)

CONSTRUCTION CASH FLOW AND RISK S-CURVES DEVELOPMENT APPROACH, AND AREA METHOD ANALYSIS AT THE PRECONSTRUCTION STAGE FROM CLIENT PERSPECTIVE IN THE UNITED ARAB EMIRATES

Journal of Entrepreneurship and Project Management, Vol. 6 No. 2 (2021), 1-37.

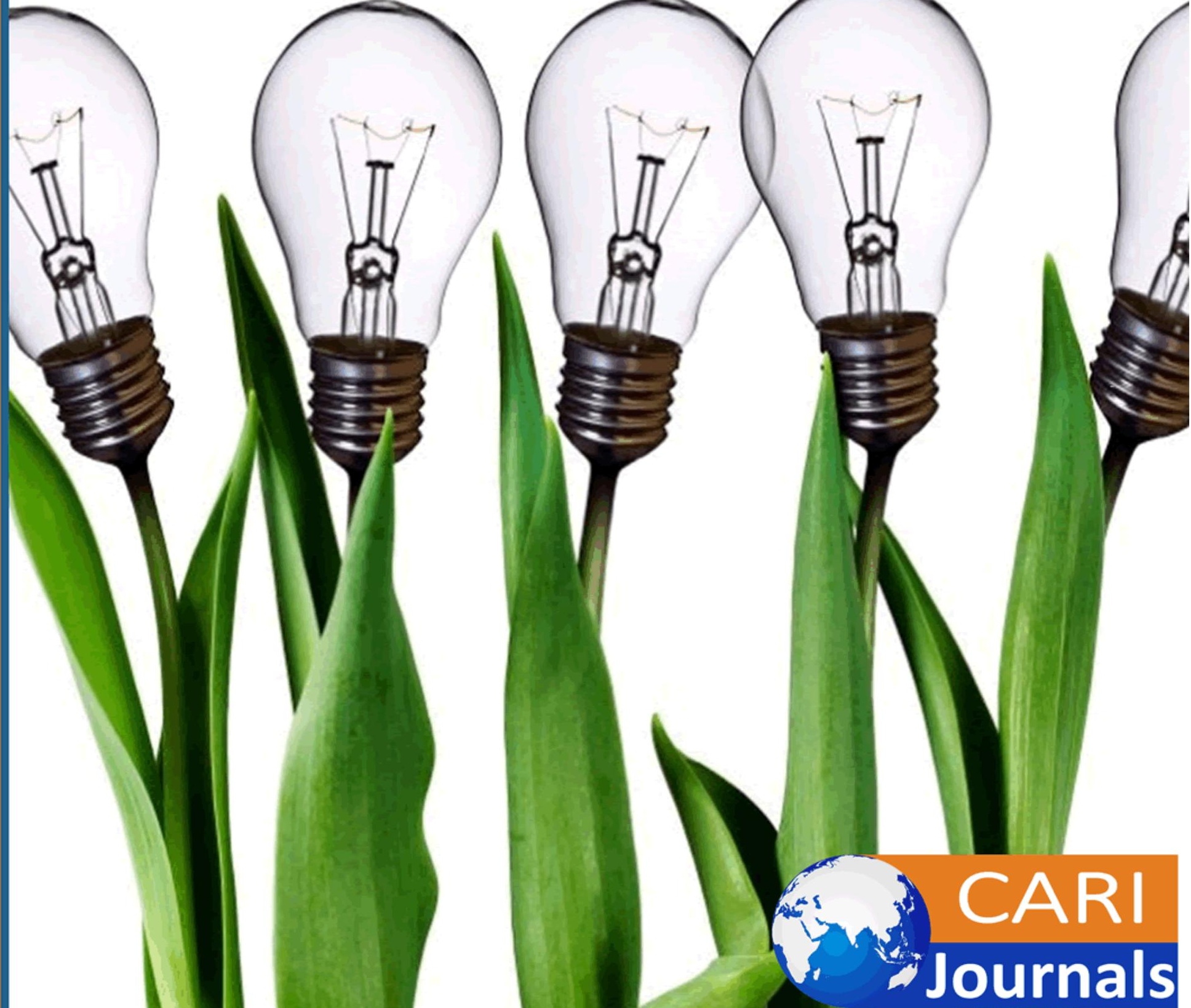




\title{
CONSTRUCTION CASH FLOW AND RISK S-CURVES DEVELOPMENT APPROACH, AND AREA METHOD ANALYSIS AT THE PRECONSTRUCTION STAGE FROM CLIENT PERSPECTIVE IN THE UNITED ARAB EMIRATES
}

\author{
Ahmed Sadek \\ The British University in Dubai \\ 2015132021@student.buid.ac.ae
}

\begin{abstract}
Purpose - Understanding construction cash flow estimation is crucial for project success. Experts are concerned about project's cash-flow and risk estimation and control. Latest construction studies concentrated on modelling and estimating construction costs and risks.

Methodology - This article aims to approach pure quantitative mathematical modelling to develop the SCurves (i.e., cash-flow and risks) and to develop the cash-flow simple area method. This research referred to the mathematical definitions of construction cash-flow and risks, integrating a clear systematic approach to develop the s-curves and to build the simple-area-method.

Findings - This research paper reviled that construction cash-flow and risk s-curves can be developed at the preconstruction stage, mathematically, without the need for having cost historical data of similar completed projects. In addition, this article has provided a simple area method approach mathematically, for construction cash flow analysis, using the basic developed cash-flow s-curve and the actual cost data of, at least, 2 completed similar projects. The simple area method is proved effective to provide a better understanding of cash-flow behaviour of the analysed projects' type. However, the s-curves development can be generalised to cover construction cost and risk simple s-curves, while the area method is restricted with the projects' characteristics (i.e., type, size, location, etc.) used in developing the simple area.

Unique contribution to theory, practice and policy - The significance of this study is to provide an SCurve development approach for both cashflow and risk percentages from client perspective at the preconstruction stage, using solely the tender contract value. And to provide a simpler stochastic area method approach for project management professionals/researchers, who do not have large amount of historical similar projects' cost data. Originality, theoretical-implications, practical-implications, and limitations are presented in the conclusion for future research.
\end{abstract}

Keywords: Construction, Cash Flow, Risk, S-Curve, Area Method Approach, Cost Estimation.

\section{INTRODUCTION}

In this article, construction cash flow and risk s-curves will be developed at the pre-construction stage following simple logistic s-curve requirements and guidelines. Then, it will develop a new construction cash flow area-method approach. This is important due to its extreme need for modelling and analysing 
costs and risks. Finally, this article will conclude with the findings and recommendation for future research.

\section{LITERATURE REVIEW}

In this part of the article, the need for further construction cost and risks research is presented systematically. It is found mandatory to deliver an approach for creating construction cost and risk s-curves behaviours at the pre-construction stage. However, the problem-statement, aim and significance are detailed to provide clear understanding of the analysis, findings and conclusion parts of this research paper.

\subsection{The Need for Construction Cost Cash-Flow and Risks Quantitative Analysis}

Although risk quantitative management and analysis are ignored, they are essential to predict its costs and impact on planned time, performance, and budget of construction projects (Poh \& Tah 2006). So, it is imperative and valuable to identify and assess possible known risks and predict the unknown risks in projects at the pre-construction stage (Schieg 2010). This will avoid cost overrun due to waste of time or resources; however, researchers proved that risk factors and variables are major and required to be considered while integrating and analysing construction cost models (Schieg 2010). For more than 40 years, these factors and variables of industrial businesses' risks are projects' concerns for project managers and operations managers (Seshadri \& Subrahmanyam 2005). However, the risks and cost statistical and mathematical equations are the best way to measure and evaluate the financial and non-financial assets' risks impact on predicting costs (Hernandez-Sancho, Molinos-Senante \& Sala-Garrido 2011). In other words, the relationship between costs and risks has been proved by researchers that costs and time impact risks; however, vice versa connection of variables' sets did research in cost estimating and modelling take into consideration risks from identification stage to assessment stage using integrated models (Doloi 2012). In 2010, researchers started to develop and compare cost modelling of constructions to reach more accurate solutions using mathematical equations for a précised value before executing projects (Petroutsatou \& Lambropoulos 2010). Researchers' models covered fuzzy techniques for reasoning and analysing construction risks to take and implement appropriate decisions (Zeng, An \& Smith 2007). These fuzzy modelling of risks opened the door for more appropriate methods and approaches to analyse risks with the other factors and categories such as system dynamics (Nasirzadeh, Khanzadi \& Rezaie 2013). Therefore, cost modelling and its interaction with risks must be considered based on the latest empirical research studies. The latest tools and results of modelling costs and risks in the construction industry, according to scientific journal publications required the highest possible inputs accuracy of the developed cost model. This is including accurate construction increasing cash-flow s-curve behaviour and accurate construction cost-risks decreasing s-curve behaviour.

After that, risks lead to extra costs in the construction industry due to improper assessment processes or misidentifying some of the critical risks; so, variables of risks are impacting estimated budget accuracy significantly when they cause changes in costs of construction execution (Ali \& Kamaruzzaman 2010). This is proving the strong connection of construction cash-flow and risks mathematically. This research paper will provide, in percentage, the behaviour of each s-curve time step for both cash-flow and cost-risks. Nevertheless, most of the time, risks are identified and assessed to provide the proper performance of the project delivery processes; thus, performance variables are considered critical projects cost modelling (Ali 
\& Kamaruzzaman 2010). According to some researchers, costs mathematical modelling in building construction projects, optimises estimated results when related to risky components such as time, waste, and energy (Abanda, Tah \& Cheung 2013). It was also proposed to model costs against an important criterion such as the cost impact of risky variables over time (Abanda, Tah \& Cheung 2013). This was done by integrating and designing a set of equations required to get better results (Abanda, Tah \& Cheung 2013). Cost modelling in public-private partnership construction projects is considered a big challenge that has to be eliminated or minimized for more accurate financing and funding without facing challengeable obstacles. However, these obstacles may lead to delay or fail projects at the execution stage (Akinyemi et al., 2009). In order to solve risky cost decisions, it was found in research that most construction practitioners and professionals such as project managers use "Cost-Effectiveness Acceptability Curve" (CEAC) for appropriate and optimum decisions. For instance, the percentage of using the (CEAC) has increased from $2.1 \%$ to $32.6 \%$ between 2001 and 2006, proving its efficiency and deep connection between risk and cost in projects (Maiwenn 2013, p. 93). This is the reason, in this article, behind using percentages of the total construction cost rather than using a cost value each timestep. Moreover, regarding construction risks and cost overrun, it is popular that structural elements and other components make people over-design the engineering component for reducing risk while increasing cost significantly; however, cost-design optimization methods have been developed by researchers to solve risk-cost conflicts between management and technical professionals (Aldwaik \& Adeli 2016). Therefore, it can be considered one construction project management cost modelling factors and variable (Aldwaik \& Adeli 2016). Also, it is supporting this research paper to provide the developed s-curves in the pre-construction stage.

Next, form investigation conducted by researchers in the private and the public sectors, it was found that estimating and predicting cost modelling must cover construction industry risks when it comes to the financial decision starting from choosing contractors until delivering the project to the end-users by the client; however, this is because of the following: 1) the underestimate at projects pre-construction stage (i.e., tendering stage), 2) the overrun of costs during projects' execution, 3) the financial and funding constraints of appearing risks and claims made by projects parties (Ayangade, Wahab, \& Alake 2009). This makes cost modelling accuracy extremely significant in the construction industry from the client's point of view (Ayangade, Wahab, \& Alake 2009). Therefore, it is essential to cover construction cash-flow and costrisks s-curve in this research modelling study.

One of the effective methods of analysing construction costs under the risks and uncertainty in developing a fuzzy approach and framework (Dikmen, Birgonul \& Han 2007; Baloi \& Price, 2003). This will help make an appropriate financial decision and estimation (Dikmen, Birgonul \& Han 2007; Baloi \& Price, 2003). The fuzzy framework has been discussed in several cost research papers and thesis. It can also be based on case-based approaches for cost estimate modelling scenarios through experience and hierarchy process combination methodology (An, Kim \& Kaug 2007). It can be achieved by developing project costs predicting reasoning efficiency analysis (An, Kim \& Kaug 2007). So, the dynamical modelling of risks in construction projects using fuzzy logic showed that system dynamics could identify and allocate risks at the early stages of executions while it can also help in eliminating unnecessary risks before experiencing its impact on costs, time, or performance (Wang, Ding, et al. 2016; Nasirzadeh, Khanzadi \& Rezaie 2014). After that, risk modelling identified different reasons behind different pricing results for the same 
construction project processes and activities in different times and positions; for example, political, law, and international procedures risks as significant in modelling costs accurately (Cohen \& Kunreuther 2007). However, it is essential to identify the most accurate costs of resources and activities during the project's stages (Eden, Williams \& Ackermann 2005; Liu, Xu et al. 2012). This can be done through the outputs on this paper, including the developed s-curves. For example, the "Vector Autoregression" Model (i.e., VAR) proved that budgeting processes for long and short-term project operations have significant impacts on CCI "Construction Cost Index" stochastic forecasting (Xu \& Moon 2013, p.10). So, the risks of changing the project schedule will significantly change its pricing, and the estimated costs and budget accuracy will change accordingly (Pajares \& López-Paredes 2011; Mizell \& Malone 2007). This is because of economics and policy conditions, which may change resource prices variations (Pajares \& López-Paredes 2011; Mizell \& Malone 2007). Therefore, the "earned value management," besides monitoring and controlling techniques, needs to be considered when scheduling processes to fix estimated budget as much as possible (Pajares \& López-Paredes 2011, p.615; Mizell \& Malone 2007).

Besides, researchers found out that it is crucial to model cost and time in mega construction projects such as tunnels (Isaksson 2002). However, the tremendous adverse effects of high risks and uncertainty of construction projects, such as infrastructure and tunnelling projects, are caused by over costs and delays resulting from an improper assessment of cost and time risks at pre-construction stages (Torp et al. 2016; Memon et al. 2010; Thijssen 2015; Isaksson 2002). These previous researches declared that cost estimation modelling is essential for megaprojects and investigated this importance validity using real projects data; therefore, this research study will investigating the applicability to create a dynamical area method with a number of projects less than 30 projects. Also, it is agreed that over costs in construction projects are happening because of several factors, including unfixed costs after fixing the budgets, finalizing tender documents, starting project's activities under inflation, which are changing actual costs from the expected budget (Isaksson 2002; Ong \& Ong 1986; Toh, Ting, et al. 2012; Peng \& Lai 2012; Zakis, Zakis \& Arfridsson 2017). So, time and cost modelling of projects are significant because of its power to shape clients and contractors' decision-makers who are the most powerful and essential stakeholders in mega projects (Isaksson 2002; Acebes et al. 2013; Signor et al. 2016; Karim \& Adeli 1999). As a result, this research study will investigate cost estimation accuracy in housing mega residential projects in the UAE. Besides, researchers concluded that research mathematical modelling and approaches, including cash-flow and risks s-curve simulating technique, is to maximize forecasting decisions accuracy (Isaksson 2002; Meyer et al. 2013; Matto \& Sippola 2016; Kleyner \& Sandborn 2008; Hamaker \& Componation 2005; Sher \& Punglia 2014; Shah \& Goldstein 2006; Lee, Lee, et al. 2011).

In other words, the advantage of including cost overrun estimate calculations and time modelling mathematically from client and contractor perspectives is to help in making better decisions regarding tender budget and pricing; however, studies found that the "construction-contracting" method can consume and waste too much time and money during project execution stage (Isaksson 2002, p.9; Hu \& He 2014; Jrade \& Lessard 2015; Wilke 2005). Overrun and over budget uncertainties are high costs risks on contractors and clients (Isaksson 2002). Cost-time modelling has been proposed based on estimation and the practical modelling of projects' cost dynamics (Isaksson 2002; Zhu et al. 2007; Adey et al. 2012; Howell $\&$ Koskela 2000). This will help choose the method of executing the project which is critical and necessary 
Journal of Entrepreneurship and Project Management

ISSN 2520-9116 (Online)

Vol. 6, Issue No.2, pp 1 -37, 2021

$\underline{\text { www.carijournals.org }}$

to determine the time and cost modelling (Isaksson 2002; Koo et al. 2010; Battistoni et al. 2016).

\subsection{Problem Statement}

Researchers and professional experts are experiencing challenges in studying the impact of variables on construction cash-low and risks. This requires establishing a scientific simple s-curves behaviour without any additional impact on it.

\subsection{Research Aim and Significance}

This article aims to provide a systematic approach to develop construction cash flow and risks s-curves. These s-curves are covering a period of 3 years, while considering only 8 timesteps each year. This will ensure keeping the privacy of the original used data of the area method, which is based on 12 timesteps (i.e., 12 month). Then, a stochastic area method approach will be provided, which can be created with lower number of projects than what is required in the previous published research (i.e., $\geq 30$ projects). This will help construction cost researchers and professionals to proceed with their analysis, although there is a challenge of having enough previous projects' data (i.e., $\leq 29$ projects). The significance of this research is to provide the equations system and approaches required for deferent types of quantitative and mixedmethod analysis. This can be used and fed into a wide range of software programmes dynamically over time. Finally, this research is providing the first stochastic cash flow area method if UAE housing residential building projects.

\section{RESEARCH METHODOLOGY}

This article is using pure mathematical equations system to develop the construction cost S-Curves behaviours (i.e., cash-flow and risks) and to establish a simple area method to estimate cash-flow behaviour. This research referred to the mathematical definitions of construction cash-flow and risks, integrating a clear systematic approach to develop the s-curves and to build the simple-area-method. The connection, between cost cash-flow and cost risks s-curves, is justified and proved mathematically to support the quantitative integration of project management future cost modelling and analysis for both researchers and industry professionals.

\section{CONSTRUCTION COST S-CURVES AND AREA METHOD DEVELOPMENT}

This article is using pure mathematical equations system to develop the construction cost S-Curves behaviours (i.e., cash-flow and risks) and to establish a simple area method to estimate cash-flow behaviour. This research referred to the mathematical definitions of construction cash-flow and risks, integrating a clear systematic approach to develop the s-curves and to build the simple-area-method. The connection, between cost cash-flow and cost risks s-curves, is justified and proved mathematically to support the quantitative integration of project management future cost modelling and analysis for both researchers and industry professionals. 


\subsection{Construction Cash Flow S-Curve Development}

Creating an s-curve in construction cost research is including three main approaches (Cristóbal 2017; Kucharavy \& Guio 2014). The first approach uses historical related projects' cost s-curve to build the estimating model's s-curve (Cristóbal 2017). This is commonly used to ensure accurate estimation of similar projects after understanding and implementing lessons learned and best practices (Cristóbal 2017; Odeyinka, Lowe \& Kaka 2013; Abanda, Tah \& Cheung 2013; Ahiaga-Dagbui 2014; Kucharavy \& Guio 2014). It will be based on measuring curve parameters from the actual project's cost curve data (Cristóbal 2017). These parameters are including 4 points to decide the behavior of desired s-curve (Cristóbal 2017). These 4 points will be substituting the $\alpha$ and T0 shown in equation 2 (Cristóbal 2017). The 4 points are extracted from the actual projects s-curve (i.e., time-cost curve) to include the data of 20\%-point, 25\%-point, 75\%-point, and $80 \%$-point (Cristóbal 2017). It is proved accurate in previous literature to draw s-curve accurate yield points and its growth (Cristóbal 2017). The second approach is by taking an s-curve equation related to the same project type of desired research and having the same duration to start estimating costs based on possible investigation scenarios (Cristóbal 2017; Odeyinka, Lowe \& Kaka 2013; Abanda, Tah \& Cheung 2013; Kucharavy \& Guio 2014). This approach is accurate in modelling the researcher's investigation topics related to cost cash flow in the construction stage (Cristóbal 2017; Odeyinka, Lowe \& Kaka 2013; Abanda, Tah \& Cheung 2013; Kucharavy \& Guio 2014). This research is modelling cash flow itself and will not completely follow previous researchers' s-curves. This is because other s-curves may have experienced factors that are not included in this research. Therefore, it may impact this research accuracy. The third approach is to have a standard simple logistic S-curve for the desired cash flow quantity over the desired project's construction duration (Kucharavy \& Guio 2014).

Past research's output equations and integration provided valid Construction Cost S-Curve as shown in equations 1 to 4 (Cristóbal 2017; Odeyinka, Lowe \& Kaka 2013; Abanda, Tah \& Cheung 2013; AhiagaDaghui 2014; Kucharavy \& Guio 2014). It agreed that the construction s-curve is following the logistic exponential s-cure shown in equation 1 (Cristóbal 2017; Odeyinka, Lowe \& Kaka 2013; Abanda, Tah \& Cheung 2013; Ahiaga-Daghui 2014; Kucharavy \& Guio 2014). Researchers developed equation 1 to equation 4 for better performance in computing construction cash flow (Cristóbal 2017; Ahiaga-Daghui 2014; Kucharavy \& Guio 2014). This will require certain requirements to be established according to Kucharavy and Guio (2014), as shown in Figure 1.

$$
f(x)=\frac{1}{1+e^{-x}}
$$

$X$ is the time point on S-Curve chart

$e$ is exponential constant.

$$
f(x)=\frac{1}{1+e^{-\propto\left(t-T_{0}\right)}}
$$

$\mathrm{T}_{\mathbf{0}}=$ The maximum slop adjusting parameter.

The maximum slop increases as it increases.

$\mathrm{t}=$ The time step of $\mathrm{f}(\mathrm{x})$ cash flow point.

$$
\left\{\begin{array}{l}
\propto\left(t_{1}-T_{0}\right)=\ln \left(\frac{1}{f_{1}}-1\right) \\
\propto\left(t_{2}-T_{0}\right)=\ln \left(\frac{1}{f_{2}}-1\right)
\end{array}\right.
$$


Journal of Entrepreneurship and Project Management

ISSN 2520-9116 (Online)

Vol. 6, Issue No.2, pp $1-37,2021$

www.carijournals.org

From similar literature:

$$
f(X)=\text { Cost } \mathrm{S}-\text { Curve }=\frac{1}{1+\mathrm{e}^{-1.22(\mathrm{t}-1.88)}}
$$

Adapting for 3 years construction from similar literature based on 8 time steps per year:

$$
f(X)=\text { Cost } \text { S-Curve }=\frac{1}{1+\mathrm{e}^{-2.7(\mathrm{t}-1.4)}}
$$

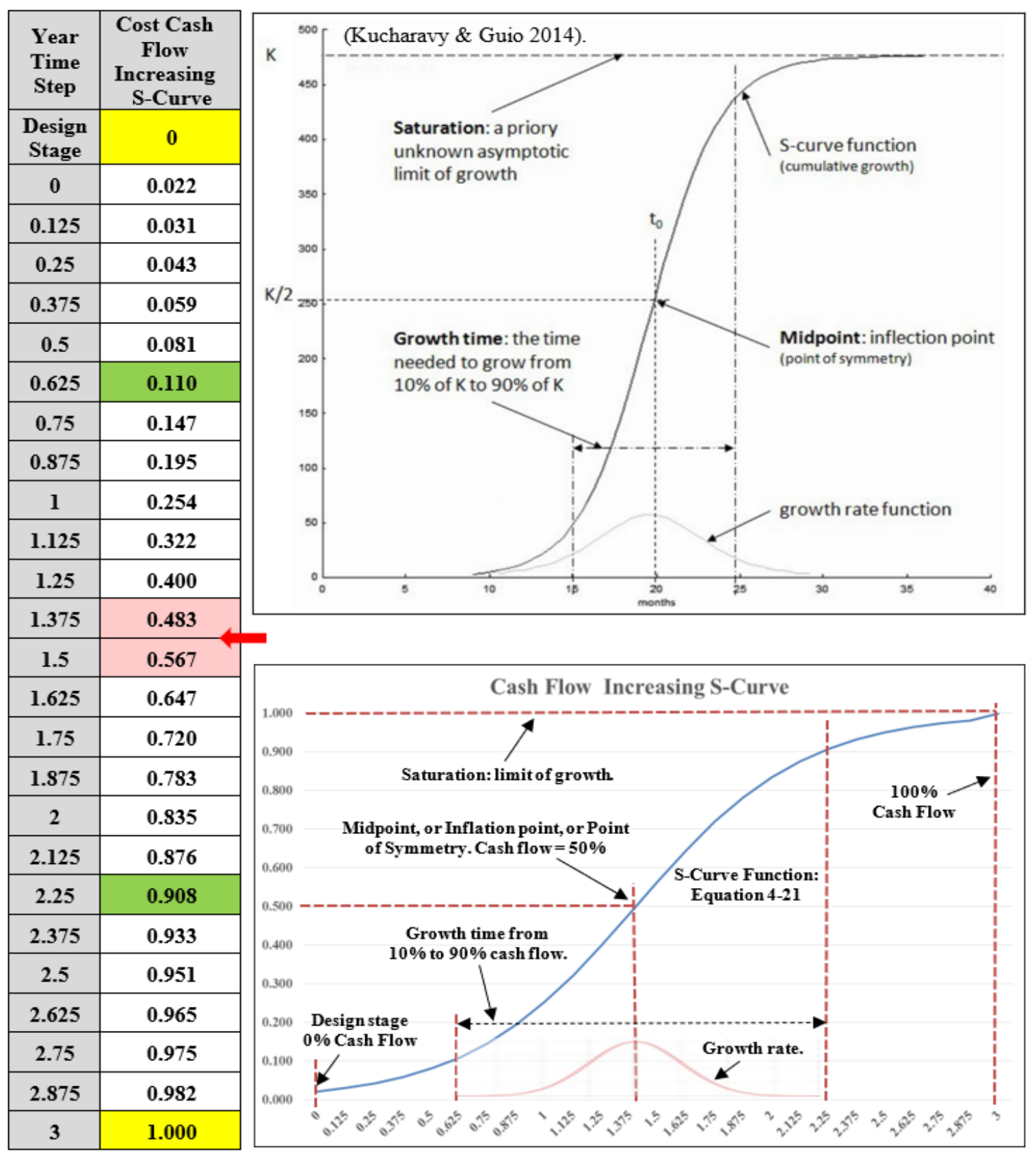

Figure 1. Adapted figure showing fitting parameters: The final 8-timesteps $S$-Curve used in this research construction cost modelling based on literature guidance (Cristóbal 2017; Kucharavy \& Guio 2014). However, K value represent construction cost of this research. 
There are three steps need to be satisfied to create a fitted simple estimating s-curve meeting actual data accuracy level (Kucharavy \& Guio 2014). First, estimating the curve lower and upper limits (Kucharavy \& Guio 2014). This research's lower limit starts from zero cash spent, and the upper limit is equal to $100 \%$ of project cash spent. Second, allocate s-curve fitting parameters, as shown in Figure 1 (Kucharavy \& Guio 2014). This will include the growth time needed for costs to increase from $10 \%$ spent to $90 \%$ spent (Kucharavy \& Guio 2014). Then, allocate the time midpoint where estimated costs reach $50 \%$ of total costs (Kucharavy \& Guio 2014). Third, examine and reduce the actual-to-estimate residuals (i.e., data-to-model residuals) to ensure that the estimated s-curve reflects the actual estimated costs (Kucharavy \& Guio 2014). Therefore, this research used previous construction equations to fit its adjustment parameter (i.e., $\alpha(t-T 0)$ ) to meet the literature's required fitting rules, as shown in Figure 1. Equation 4 is the final used equation is the cash flow S-Curve Model.

\subsection{Construction Risk S-Curve Development}

According to Oxford Advanced Learner's Dictionary (1995), risk definition is the possibility of suffering harm or loss; or facing danger or failure (Baloi \& Price 2003). Therefore, it is challenging to generalize its measurement or its behaviour throughout projects. This mathematical modelling is deterministic because its answer has exact values. However, risks can be used for the study as long its definition can be met and satisfied through the assigned measurement or behaviour criteria (Baloi \& Price 2003). Several facts need to be considered in order to finalize the construction risk equation. First, construction risk is taking an exponential function for costs and financial situations (Dowd \& Cotter 2007). Second, construction (i.e., buildings) risk behaviour is based on a decreasing S-Curve (Pajares \& López-Paredes 2011). Third, to satisfy the risk definition, the probability of facing danger during construction will be in the remaining time, and the amount of cash paid. These are the required risk behaviour considerations in this research cost estimation model. The conditional probability intersection relationship between two events is mathematically represented by multiplication (Techet 2005). There are two calculation parts of construction cost risks. The cash flow s-curve is based on time and costs ratio/percentage (i.e., $\mathrm{X}$ and $\mathrm{Y}$ ). First, the probability of facing danger in time at each time step is the weightage of remaining time concerning total construction time. It will equal the ratio of time remaining to total construction time, as shown in equation 5. Second, the probability of facing danger in costs at each time step is the weightage of remaining costs concerning total construction cost. It will equal the ratio of remaining unpaid value to total construction cost. This can be extracted from Figure 2 and equations 6 and 7. In equation 8, limits function are important to show the exact calculations of CS as risk time step value (i.e., TS) approaches cash flow s-curve time step value (i.e., t). Construction risks are active from just before the first cash flow payment until the last cash flow payment. In other words, construction risks are $100 \%$ just before starting and $0 \%$ at the finishing time. Thus, the construction risk curve will equal the probability of facing risk in the remaining time multiply by the probability of facing danger in the remaining cash flow amount will equal to this research risk decreasing s-curve as shown in equations 8 and 9 (Pajares \& López-Paredes 2010). Therefore, this research risk s-curve will equal to Figure 2 chart values. The probability of facing risk in remaining time multiply by the probability of facing danger in remaining cash flow will be this research Risk S-Curve as shown in equation 5 to 9 and Figure 2 (Pajares \& López-Paredes 2010; Techet 2005; Dowd \& Cotter 2007). 
Journal of Entrepreneurship and Project Management

ISSN 2520-9116 (Online)

Vol. 6, Issue No.2, pp 1 -37, 2021

$\boldsymbol{P}_{T}=\frac{\boldsymbol{T}_{E}-\boldsymbol{T}_{S}}{\boldsymbol{T}_{\boldsymbol{E}}}=$ Time Risk

$\boldsymbol{P}_{T}$ is the probability of time danger.

$\mathbf{T}_{\mathbf{E}}$ is the total execution time.

$\mathbf{T}_{\mathrm{s}}$ is the risk time step value.

$C_{S}=\lim _{\mathrm{T}_{S} \rightarrow \mathrm{t}}\left(\frac{1}{1+\mathrm{e}^{-1.22(\mathrm{t}-1.88)}}\right)$

$\mathrm{C}_{\mathbf{S}}$ is the cost $\mathrm{S}$ curve value at each time step (from 0 to 1 ).

t is the time step of $\mathrm{f}(\mathrm{x})$ cash flow point.

$P_{C}=\left[1-C_{S}\right]$

$\boldsymbol{P}_{\boldsymbol{C}}$ is the probability of cost danger.

$P($ Time Risk $\cap$ Cost Risk $)=P_{T} * P_{C}$

Risk S-Curve $=\frac{T_{E}-T_{S}}{T_{E}} *\left(1-\lim _{T_{S} \rightarrow \mathrm{t}}\left(\frac{1}{1+\mathrm{e}^{-1.22(\mathrm{t}-1.88)}}\right)\right)$

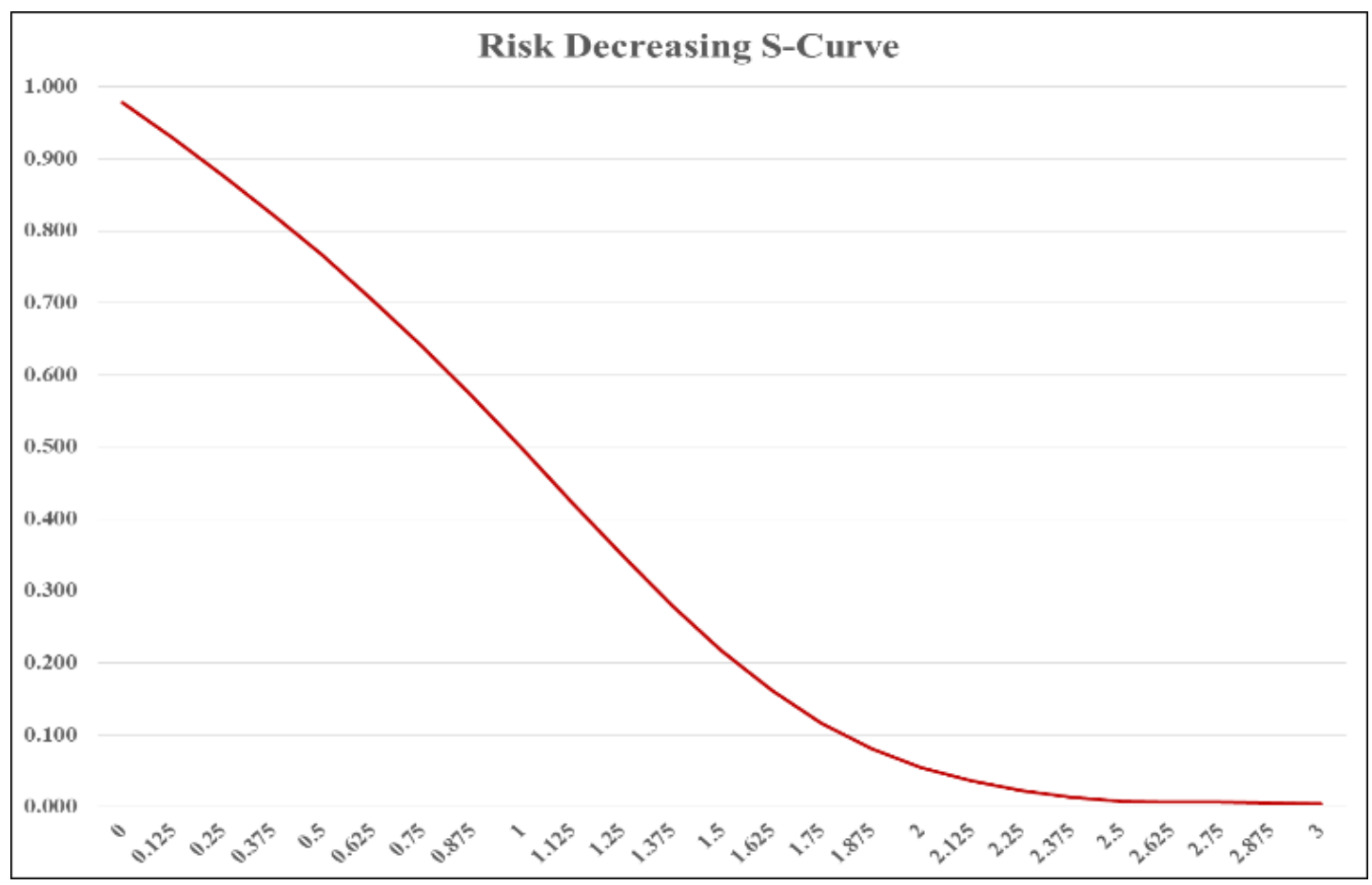

Figure 2. Trending 8-timesteps construction Risk decreasing S-Curve. 


\subsection{Cash Flow and Risk S-Curves for 1 year to 5 years Construction Periods}

In this part of the article, cash flow and risks s-curves are provided for construction phase including two scenarios. First, the construction stage which is ending by the project completion. This is an appropriate curve if the cost analysis is considering the retention bond instead of holding the actual cash during the defect notification period (DNP) / the defect liability period (DLP). Or, in case if the researcher / professional experts are investigating the cash flow of a project's whole life cycle; however, in this scenario, the operational expenditures will start directly after the project's completion and the retention amount will merge with the operational costs. Therefore, it is better to consider the retention behaviour like the retention bond behaviour of the construction phase cash flow s-curve. Retention bonds have been defined as an alternative process of the keeping the retention cash during the DNP/DLP stage (RICS 2012).

In figures 1 and 2, the 8-timesteps curves will be used in the following parts of creating the cost area method analysis. Nevertheless, tables 1 to 10 and figures 13 to 22, in the research paper are detailing the scurves based on 12-timesteps for direct use by cost analysts. Both cash flow and risks s-curves are based on the perfect cash flow s-curves, which is following Kucharavy \& Guio (2014) s-curves guidance.

Next, it is important to detail the s-curves tables' colour coding to understand how the provided data and plotted charts are satisfying the published s-curve criteria. Table 1is detailing the meaning of each colour code used in tables 2 to 11 .

Table 1. Identifying the colour coding used in tables 2 to 11.

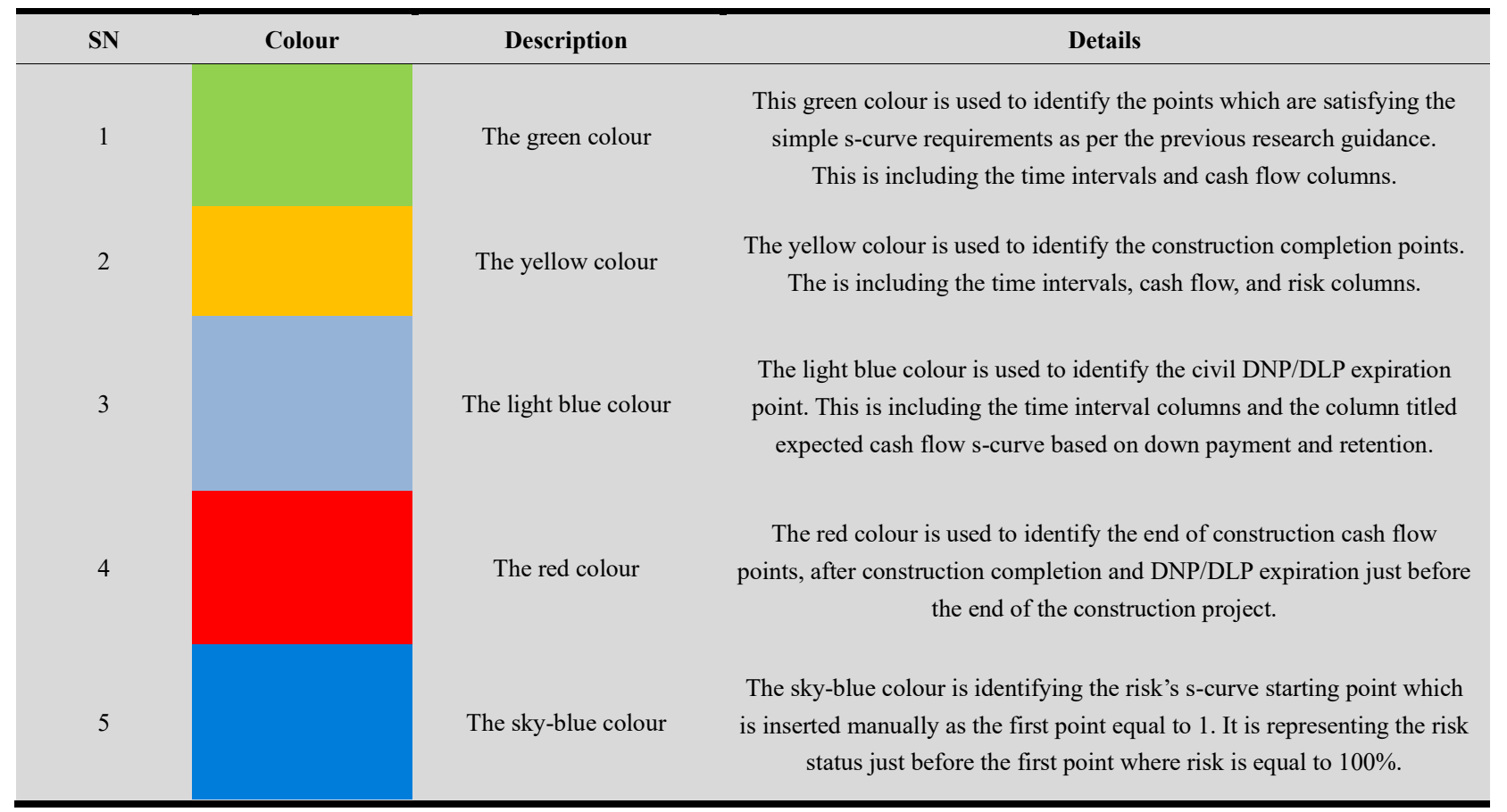


Journal of Entrepreneurship and Project Management

ISSN 2520-9116 (Online)

Vol. 6, Issue No.2, pp 1 -37, 2021

www.carijournals.org

Table 2. Identifying 1 year construction Cash flow and Risk S-Curves based on accepting retention bond.

\begin{tabular}{|c|c|c|c|c|c|}
\hline \multirow{2}{*}{$\begin{array}{l}\text { Time } \\
\text { Label }\end{array}$} & \multirow{2}{*}{$\begin{array}{l}\text { Month Interval } \\
\text { (n) }\end{array}$} & Year Interval & $\begin{array}{c}\text { Expected Cash Flow S-Curve } \\
\text { Based on Down payment \& } \\
\text { Retention }\end{array}$ & Perfect Cash Flow S-Curve & Risk S-Curve \\
\hline & & \begin{tabular}{|c|} 
"Month Interval $(\mathbf{n}) " *$ \\
$(1 /$ "\# of time steps in a \\
year (i.e., 12)")
\end{tabular} & $\begin{array}{l}\text { 1/(1+(EXP(-5.7*("Year } \\
\text { Interval"-0.5)))) }\end{array}$ & $\begin{array}{l}\text { 1/(1+(EXP(-10.6*("Year } \\
\text { Interval"-0.5)))) }\end{array}$ & $\begin{array}{c}\text { ((1 -"Year Interval (n-1)")/1)* } \\
\text { (1-"Perfect Cash Flow S-Curve (n-1)") }\end{array}$ \\
\hline Start & 0 & \begin{tabular}{|l|}
0.00 \\
\end{tabular} & 0.05 & 0.00 & 1.000 \\
\hline Month 1 & 1 & 0.08 & 0.09 & 0.01 & 0.995 \\
\hline Month 2 & 2 & 0.17 & 0.13 & 0.03 & 0.906 \\
\hline Month 3 & 3 & 0.25 & 0.19 & 0.07 & 0.810 \\
\hline Month 4 & 4 & 0.33 & 0.28 & 0.15 & 0.701 \\
\hline Month 5 & 5 & 0.42 & 0.38 & 0.29 & 0.570 \\
\hline Month 6 & 6 & 0.50 & 0.50 & 0.50 & 0.413 \\
\hline Month 7 & 7 & 0.58 & 0.62 & 0.71 & 0.250 \\
\hline Month 8 & 8 & 0.67 & 0.72 & 0.85 & 0.122 \\
\hline Month 9 & 9 & 0.75 & 0.81 & 0.93 & 0.049 \\
\hline Month 10 & 10 & 0.83 & 0.87 & 0.97 & 0.017 \\
\hline Month 11 & 11 & 0.92 & 0.91 & 0.99 & 0.005 \\
\hline Month 12 & 12 & 1.00 & 0.95 & 1.00 & 0.001 \\
\hline & Project & End & 1.00 & 1.00 & 0.000 \\
\hline
\end{tabular}

Expected, 1 year Construction, Cash Flow S-Curve

Based on Down payment \& Retention

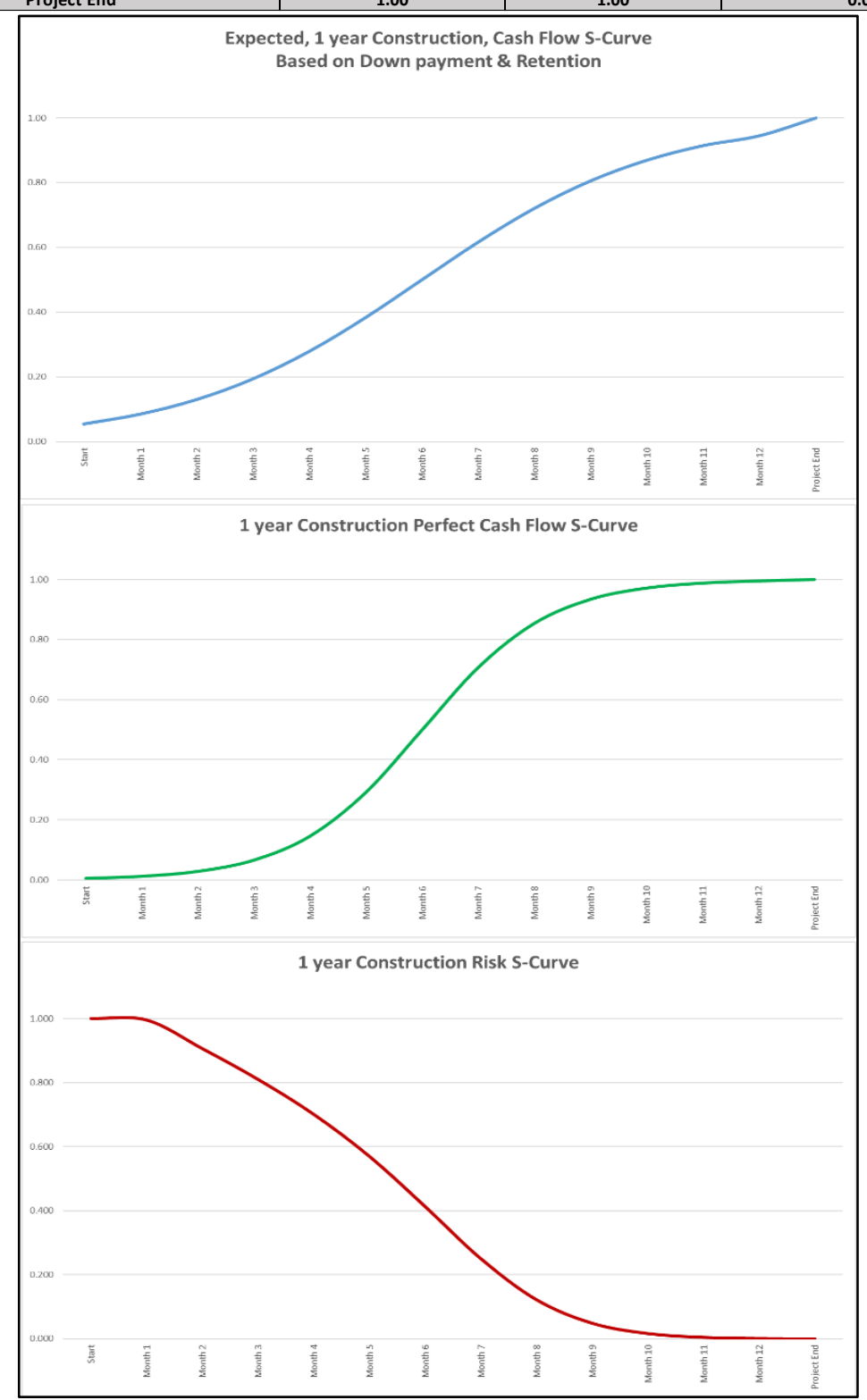

Figure 3. Trending 1 year construction Cash flow and Risk S-Curves based on accepting retention bond. 
Journal of Entrepreneurship and Project Management

ISSN 2520-9116 (Online)

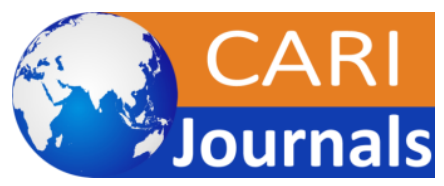

Vol. 6, Issue No.2, pp $1-37,2021$

www.carijournals.org

Table 3. Identifying 1 year construction Cash flow and Risk S-Curves based on DNP/DLP without retention bond.

\begin{tabular}{|c|c|c|c|c|c|}
\hline \multirow{2}{*}{$\begin{array}{l}\text { Time } \\
\text { Label }\end{array}$} & \multirow{2}{*}{$\begin{array}{l}\text { Month Interval } \\
\text { (n) }\end{array}$} & Year Interval & $\begin{array}{c}\text { Expected Cash Flow S-Curve } \\
\text { Based on Down payment \& } \\
\text { Retention }\end{array}$ & Perfect Cash Flow S-Curve & Risk S-Curve \\
\hline & & $\begin{array}{c}\text { "Month Interval }(n) " * \\
\text { (1/ "\# of time steps in a } \\
\text { year (i.e., 12)") }\end{array}$ & $\begin{array}{l}\text { 1/(1+(EXP }(-5.7 *(" Y e a r \\
\text { Interval"-0.5)))) }\end{array}$ & $\begin{array}{l}\text { 1/(1+(EXP(-10.6*("Year } \\
\text { Interval"-0.5)))) }\end{array}$ & $\begin{array}{c}\left(\left(1-" \text {-"Year Interval }(n-1)^{\prime \prime)}\right) / 1\right)^{*} \\
(1-" P e r f e c t \text { Cash Flow S-Curve (n-1)") }\end{array}$ \\
\hline Start & 0 & 0.00 & 0.05 & 0.00 & 1.000 \\
\hline Month 1 & 1 & 0.08 & 0.09 & 0.01 & 0.995 \\
\hline Month 2 & 2 & 0.17 & 0.13 & 0.03 & 0.906 \\
\hline Month 3 & 3 & 0.25 & 0.19 & 0.07 & 0.810 \\
\hline Month 4 & 4 & 0.33 & 0.28 & 0.15 & 0.701 \\
\hline Month 5 & 5 & 0.42 & 0.38 & 0.29 & 0.570 \\
\hline Month 6 & 6 & 0.50 & 0.50 & 0.50 & 0.413 \\
\hline Month 7 & 7 & 0.58 & 0.62 & 0.71 & 0.250 \\
\hline Month 8 & 8 & 0.67 & 0.72 & 0.85 & 0.122 \\
\hline Month 9 & 9 & 0.75 & 0.81 & 0.93 & 0.049 \\
\hline Month 10 & 10 & 0.83 & 0.87 & 0.97 & 0.017 \\
\hline Month 11 & 11 & 0.92 & 0.91 & 0.99 & 0.005 \\
\hline Month 12 & 12 & 1.00 & 0.95 & 1.00 & 0.001 \\
\hline Month 13 & 13 & 1.08 & 0.95 & 1.00 & 0.000 \\
\hline Month 14 & 14 & 1.17 & 0.95 & 1.00 & 0.000 \\
\hline Month 15 & 15 & 1.25 & 0.95 & 1.00 & 0.000 \\
\hline Month 16 & 16 & 1.33 & 0.95 & 1.00 & 0.000 \\
\hline Month 17 & 17 & 1.42 & 0.95 & 1.00 & 0.000 \\
\hline Month 18 & 18 & 1.50 & 0.95 & 1.00 & 0.000 \\
\hline Month 19 & 19 & 1.58 & 0.95 & 1.00 & 0.000 \\
\hline Month 20 & 20 & 1.67 & 0.95 & 1.00 & 0.000 \\
\hline Month 21 & 21 & 1.75 & 0.95 & 1.00 & 0.000 \\
\hline Month 22 & 22 & 1.83 & 0.95 & 1.00 & 0.000 \\
\hline Month 23 & 23 & 1.92 & 0.95 & 1.00 & 0.000 \\
\hline Month 24 & 24 & 2.00 & 0.99 & 1.00 & 0.000 \\
\hline Month 25 & 25 & 2.08 & 0.99 & 1.00 & 0.000 \\
\hline Month 26 & 26 & 2.17 & 0.99 & 1.00 & 0.000 \\
\hline Month 27 & 27 & 2.25 & 0.99 & 1.00 & 0.000 \\
\hline Month 28 & 28 & 2.33 & 0.99 & 1.00 & 0.000 \\
\hline Month 29 & 29 & 2.42 & 0.99 & 1.00 & 0.000 \\
\hline Month 30 & 30 & 2.50 & 0.99 & 1.00 & 0.000 \\
\hline Month 31 & 31 & 2.58 & 0.99 & 1.00 & 0.000 \\
\hline Month 32 & 32 & 2.67 & 0.99 & 1.00 & 0.000 \\
\hline Month 33 & 33 & 2.75 & 0.99 & 1.00 & 0.000 \\
\hline Month 34 & 34 & 2.83 & 0.99 & 1.00 & 0.000 \\
\hline Month 35 & 35 & 2.92 & 0.99 & 1.00 & 0.000 \\
\hline Month 36 & 36 & 3.00 & 0.99 & 1.00 & 0.000 \\
\hline Month 37 & 37 & 3.08 & 0.99 & 1.00 & 0.000 \\
\hline Month 38 & 38 & 3.17 & 0.99 & 1.00 & 0.000 \\
\hline Month 39 & 39 & 3.25 & 0.99 & 1.00 & 0.000 \\
\hline Month 40 & 40 & 3.33 & 0.99 & 1.00 & 0.000 \\
\hline Month 41 & 41 & 3.42 & 0.99 & 1.00 & 0.000 \\
\hline Month 42 & 42 & 3.50 & 0.99 & 1.00 & 0.000 \\
\hline Month 43 & 43 & 3.58 & 0.99 & 1.00 & 0.000 \\
\hline Month 44 & 44 & 3.67 & 0.99 & 1.00 & 0.000 \\
\hline Month 45 & 45 & 3.75 & 0.99 & 1.00 & 0.000 \\
\hline Month 46 & 46 & 3.83 & 0.99 & 1.00 & 0.000 \\
\hline Month 47 & 47 & 3.92 & 0.99 & 1.00 & 0.000 \\
\hline Month 48 & 48 & 4.00 & 1.00 & 1.00 & 0.000 \\
\hline & Project & End & 1.00 & 1.00 & 0.000 \\
\hline
\end{tabular}
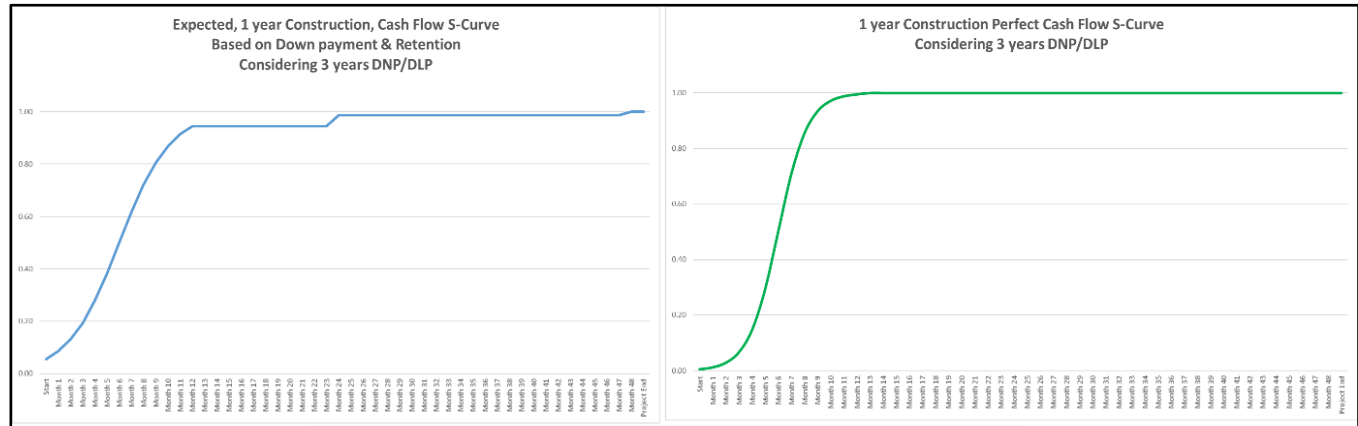

1 year Construction Risk S-Curve
Considering 3 years DNP/DLP

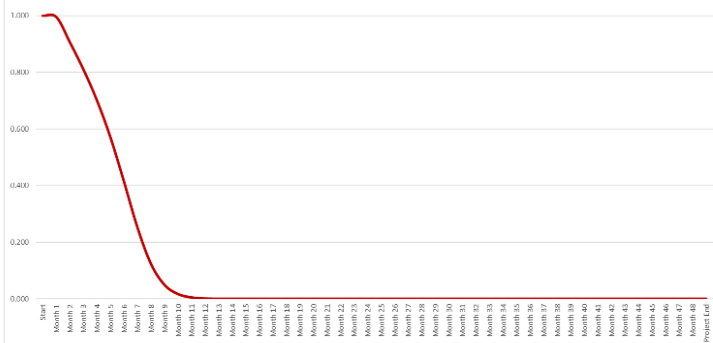

Figure 4. Trending 1 year construction Cash flow and Risk S-Curves based on DNP/DLP without retention bond. 
Journal of Entrepreneurship and Project Management

ISSN 2520-9116 (Online)

Vol. 6, Issue No.2, pp 1 -37, 2021

www.carijournals.org

Table 4. Identifying 2 years construction Cash flow and Risk S-Curves based on accepting retention bond.

\begin{tabular}{|c|c|c|c|c|c|}
\hline \multirow{2}{*}{$\begin{array}{l}\text { Time } \\
\text { Label }\end{array}$} & \multirow{2}{*}{$\begin{array}{l}\text { Month Interval } \\
\text { (n) }\end{array}$} & Year Interval & $\begin{array}{c}\text { Expected Cash Flow S-Curve } \\
\text { Based on Down payment \& } \\
\text { Retention }\end{array}$ & Perfect Cash Flow S-Curve & Risk S-Curve \\
\hline & & $\begin{array}{c}\text { "Month Interval (n)" * } \\
\text { (1/ "\# of time steps in a } \\
\text { year (i.e., 12)") }\end{array}$ & $\begin{array}{l}\text { 1/(1+(EXP(-3*("Year } \\
\text { Interval"-1)))) }\end{array}$ & $\begin{array}{c}\text { 1/(1+(EXP }\left(-5.4^{*}(" \text { Year }\right. \\
\text { Interval"-1)))) }\end{array}$ & $\begin{array}{c}\text { ((2 -"Year Interval (n-1)")/2)* } \\
\text { (1-"Perfect Cash Flow S-Curve (n-1)") }\end{array}$ \\
\hline Start & 0 & 0.00 & 0.05 & 0.00 & 1.000 \\
\hline Month 1 & 1 & 0.08 & 0.06 & 0.01 & 0.996 \\
\hline Month 4 & 4 & 0.33 & 0.12 & 0.03 & 0.860 \\
\hline Month 5 & 5 & 0.42 & 0.15 & 0.04 & 0.811 \\
\hline Month 6 & 6 & 0.50 & 0.18 & 0.06 & 0.759 \\
\hline Month 7 & 7 & 0.58 & 0.22 & 0.10 & 0.703 \\
\hline Month 8 & 8 & 0.67 & 0.27 & 0.14 & 0.641 \\
\hline Month 9 & 9 & 0.75 & 0.32 & 0.21 & 0.572 \\
\hline Month 14 & 14 & 1.17 & 0.62 & 0.71 & 0.179 \\
\hline Month 15 & 15 & 1.25 & 0.68 & 0.79 & 0.121 \\
\hline Month 16 & 16 & 1.33 & 0.73 & 0.86 & 0.077 \\
\hline Month 17 & 17 & 1.42 & 0.78 & 0.90 & 0.047 \\
\hline Month 18 & 18 & 1.50 & 0.82 & 0.94 & 0.028 \\
\hline Month 19 & 19 & 1.58 & 0.85 & 0.96 & 0.016 \\
\hline Month 20 & 20 & 1.67 & 0.88 & 0.97 & 0.009 \\
\hline Month 21 & 21 & 1.75 & 0.90 & 0.98 & 0.004 \\
\hline Month 22 & 22 & 1.83 & 0.92 & 0.99 & 0.002 \\
\hline Month 23 & 23 & 1.92 & 0.94 & 0.99 & 0.001 \\
\hline Month 24 & 24 & 2.00 & 0.95 & 1.00 & 0.000 \\
\hline
\end{tabular}

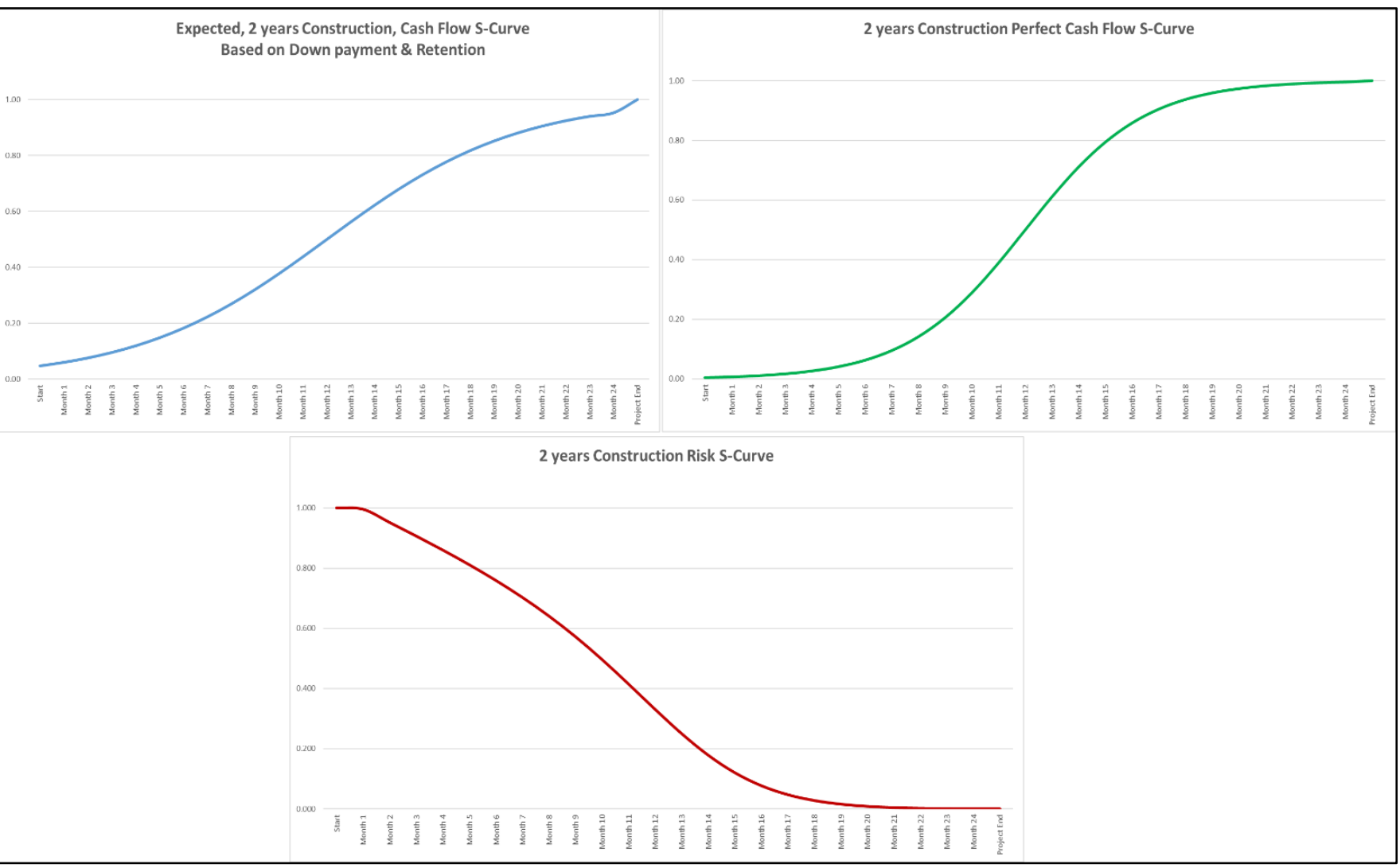

Figure 5. Trending 2 years construction Cash flow and Risk S-Curves based on accepting retention bond. 
Journal of Entrepreneurship and Project Management

ISSN 2520-9116 (Online)

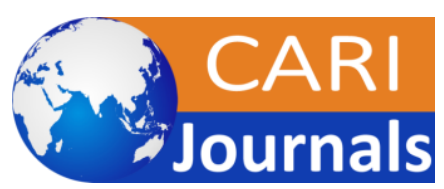

Vol. 6, Issue No.2, pp 1 -37, 2021

www.carijournals.org

Table 5. Identifying 2 years construction Cash flow and Risk S-Curves based on DNP/DLP without retention bond.

\begin{tabular}{|c|c|c|c|c|c|}
\hline \multirow{2}{*}{$\begin{array}{l}\text { Time } \\
\text { Label }\end{array}$} & \multirow{2}{*}{$\begin{array}{l}\text { Month Interval } \\
\text { (n) }\end{array}$} & Year Interval & $\begin{array}{c}\text { Expected Cash Flow S-Curve } \\
\text { Based on Down payment \& } \\
\text { Retention }\end{array}$ & Perfect Cash Flow S-Curve & Risk S-Curve \\
\hline & & \begin{tabular}{|c|} 
"Month Interval $(\mathrm{n}) " *$ \\
(1/ "\# of time steps in a \\
year (i.e., 12)")
\end{tabular} & $\begin{array}{c}1 /\left(1+\left(\operatorname{EXP}\left(-3^{*}(" Y e a r \text { Interval" }\right.\right.\right. \\
1))))\end{array}$ & $\begin{array}{l}\text { 1/(1+(EXP(-5.4*("Year } \\
\text { Interval"-1)))) }\end{array}$ & $\begin{array}{c}\left(\left(2 \text {-"Year Interval }(n-1)^{\prime \prime}\right) / 2\right)^{*} \\
\text { (1-"Perfect Cash Flow S-Curve (n-1)") }\end{array}$ \\
\hline Start & 0 & 0.00 & 0.05 & 0.00 & 1.000 \\
\hline Month 1 & 1 & 0.08 & 0.06 & 0.01 & 0.996 \\
\hline Month 2 & 2 & 0.17 & 0.08 & 0.01 & 0.952 \\
\hline Month 3 & 3 & 0.25 & 0.10 & 0.02 & 0.907 \\
\hline Month 4 & 4 & 0.33 & 0.12 & 0.03 & 0.860 \\
\hline Month 5 & 5 & 0.42 & 0.15 & 0.04 & 0.811 \\
\hline Month 6 & 6 & 0.50 & 0.18 & 0.06 & 0.759 \\
\hline Month 7 & 7 & 0.58 & 0.22 & 0.10 & 0.703 \\
\hline Month 8 & 8 & 0.67 & 0.27 & 0.14 & 0.641 \\
\hline Month 9 & 9 & 0.75 & 0.32 & 0.21 & 0.572 \\
\hline Month 10 & 10 & 0.83 & 0.38 & 0.29 & 0.497 \\
\hline Month 11 & 11 & 0.92 & 0.44 & 0.39 & 0.415 \\
\hline Month 12 & 12 & 1.00 & 0.50 & 0.50 & 0.331 \\
\hline Month 13 & 13 & 1.08 & 0.56 & 0.61 & 0.250 \\
\hline Month 14 & 14 & 1.17 & 0.62 & 0.71 & 0.179 \\
\hline Month 15 & 15 & 1.25 & 0.68 & 0.79 & 0.121 \\
\hline Month 16 & 16 & 1.33 & 0.73 & 0.86 & 0.077 \\
\hline Month 17 & 17 & 1.42 & 0.78 & 0.90 & 0.047 \\
\hline Month 18 & 18 & 1.50 & 0.82 & 0.94 & 0.028 \\
\hline Month 19 & 19 & 1.58 & 0.85 & 0.96 & 0.016 \\
\hline Month 20 & 20 & 1.67 & 0.88 & 0.97 & 0.009 \\
\hline Month 21 & 21 & 1.75 & 0.90 & 0.98 & 0.004 \\
\hline Month 22 & 22 & 1.83 & 0.92 & 0.99 & 0.002 \\
\hline Month 23 & 23 & 1.92 & 0.94 & 0.99 & 0.001 \\
\hline Month 24 & 24 & 2.00 & 0.95 & 1.00 & 0.000 \\
\hline Month 25 & 25 & 2.08 & 0.95 & 1 & 0.000 \\
\hline Month 26 & 26 & 2.17 & 0.95 & 1 & 0.000 \\
\hline Month 27 & 27 & 2.25 & 0.95 & 1 & 0.000 \\
\hline Month 28 & 28 & 2.33 & 0.95 & 1 & 0.000 \\
\hline Month 29 & 29 & 2.42 & 0.95 & 1 & 0.000 \\
\hline Month 30 & 30 & 2.50 & 0.95 & 1 & 0.000 \\
\hline Month 31 & 31 & 2.58 & 0.95 & 1 & 0.000 \\
\hline Month 32 & 32 & 2.67 & 0.95 & 1 & 0.000 \\
\hline Month 33 & 33 & 2.75 & 0.95 & 1 & 0.000 \\
\hline Month 34 & 34 & 2.83 & 0.95 & 1 & 0.000 \\
\hline Month 35 & 35 & 2.92 & 0.95 & 1 & 0.000 \\
\hline Month 36 & 36 & 3.00 & 0.99 & 1 & 0.000 \\
\hline Month 37 & 37 & 3.08 & 0.99 & 1 & 0.000 \\
\hline Month 38 & 38 & 3.17 & 0.99 & 1 & 0.000 \\
\hline Month 39 & 39 & 3.25 & 0.99 & 1 & 0.000 \\
\hline Month 40 & 40 & 3.33 & 0.99 & 1 & 0.000 \\
\hline Month 41 & 41 & 3.42 & 0.99 & 1 & 0.000 \\
\hline Month 42 & 42 & 3.50 & 0.99 & 1 & 0.000 \\
\hline Month 43 & 43 & 3.58 & 0.99 & 1 & 0.000 \\
\hline Month 44 & 44 & 3.67 & 0.99 & 1 & 0.000 \\
\hline Month 45 & 45 & 3.75 & 0.99 & 1 & 0.000 \\
\hline Month 46 & 46 & 3.83 & 0.99 & 1 & 0.000 \\
\hline Month 47 & 47 & 3.92 & 0.99 & 1 & 0.000 \\
\hline Month 48 & 48 & 4.00 & 0.99 & 1 & 0.000 \\
\hline Month 49 & 49 & 4.08 & 0.99 & 1 & 0.000 \\
\hline Month 50 & 50 & 4.17 & 0.99 & 1 & 0.000 \\
\hline Month 51 & 51 & 4.25 & 0.99 & 1 & 0.000 \\
\hline Month 52 & 52 & 4.33 & 0.99 & 1 & 0.000 \\
\hline Month 53 & 53 & 4.41 & 0.99 & 1 & 0.000 \\
\hline Month 54 & 54 & 4.50 & 0.99 & 1 & 0.000 \\
\hline Month 55 & 55 & 4.58 & 0.99 & 1 & 0.000 \\
\hline Month 56 & 56 & 4.66 & 0.99 & 1 & 0.000 \\
\hline Month 57 & 57 & 4.75 & 0.99 & 1 & 0.000 \\
\hline Month 58 & 58 & 4.83 & 0.99 & 1 & 0.000 \\
\hline Month 59 & 59 & 4.91 & 0.99 & 1 & 0.000 \\
\hline Month 60 & 60 & 5.00 & 1.00 & 1 & 0.000 \\
\hline & Project & ind & 1.00 & 1 & 0.000 \\
\hline
\end{tabular}


Journal of Entrepreneurship and Project Management

ISSN 2520-9116 (Online)

Vol. 6, Issue No.2, pp 1 -37, 2021

WwW.carijournals.org

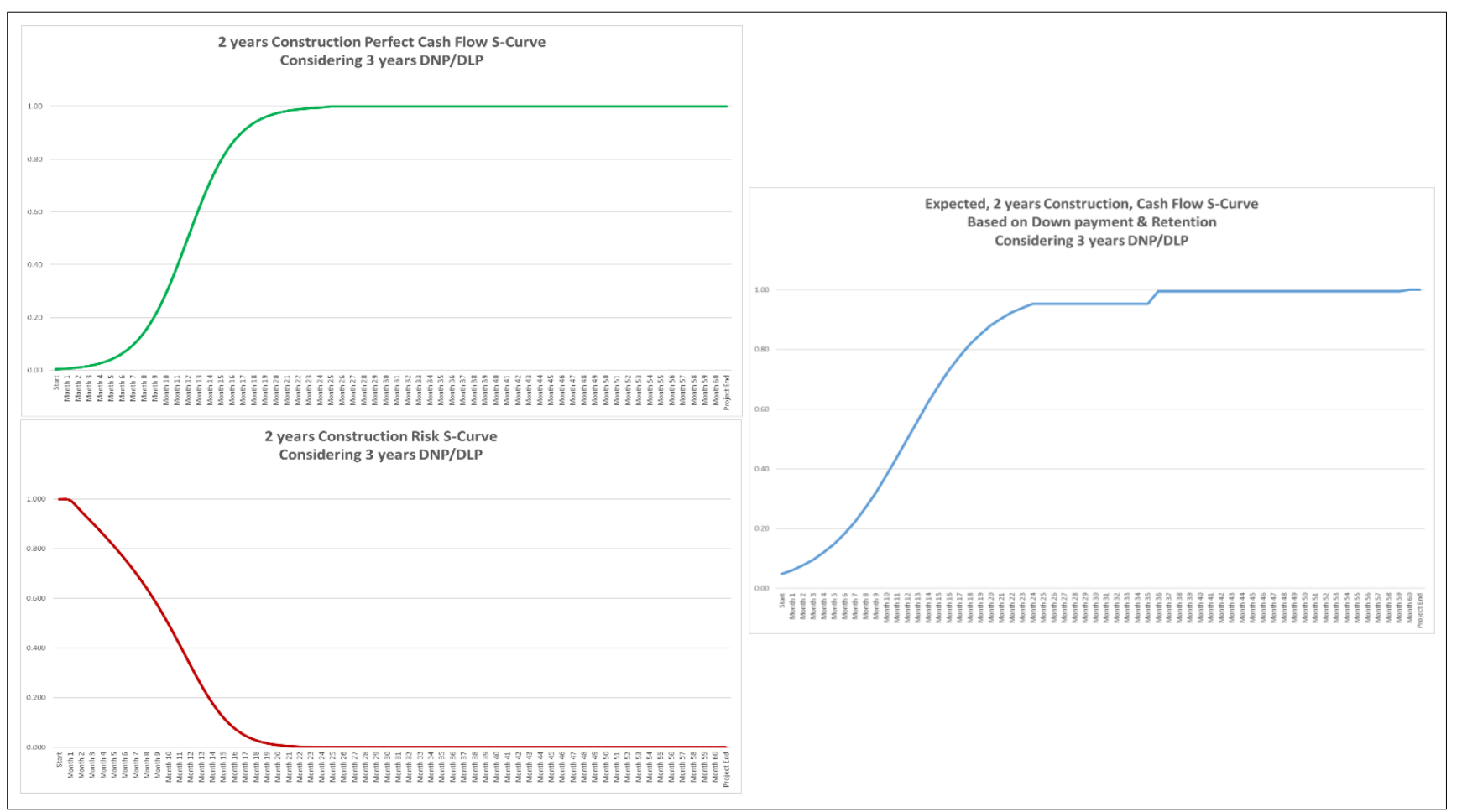

Figure 6. Trending 2 years construction Cash flow and Risk S-Curves based on DNP/DLP without retention bond.

Table 6. Identifying 3 years construction Cash flow and Risk S-Curve based on accepting retention bond.

\begin{tabular}{|c|c|c|c|c|c|}
\hline \multirow{2}{*}{$\begin{array}{l}\text { Time } \\
\text { Label }\end{array}$} & \multirow{2}{*}{$\begin{array}{c}\text { Month } \\
\text { Interval (n) }\end{array}$} & \multirow[b]{2}{*}{\begin{tabular}{|c|} 
"Month Interval $(n) " ~ *$ \\
(1/ "\# of time steps in a \\
year (i.e., 12)")
\end{tabular}} & \multirow{2}{*}{$\begin{array}{c}\begin{array}{c}\text { Expected Cash Flow S-Curve } \\
\text { Based on Down payment \& } \\
\text { Retention }\end{array} \\
1 /(1+(\text { EXP(-2*("Year } \\
\text { Interval"-1.5)))) }\end{array}$} & \multirow{2}{*}{\begin{tabular}{|c|} 
Perfect Cash Flow S-Curve \\
$\begin{array}{c}\text { 1/(1+(EXP(-3.68*("Year } \\
\text { Interval"-1.5)))) }\end{array}$ \\
\end{tabular}} & \multirow{2}{*}{$\begin{array}{c}\text { Risk S-Curve } \\
((3-" Y e a r \text { Interval }(n-1) ") / 3)^{*} \\
(1-" P e r f e c t \text { Cash Flow S-Curve (n-1)") }\end{array}$} \\
\hline & & & & & \\
\hline Start & 0 & 0.00 & 0.05 & 0.00 & 1.000 \\
\hline Month 1 & 1 & 0.08 & 0.06 & 0.01 & 0.996 \\
\hline Month 2 & 2 & 0.17 & 0.06 & 0.01 & 0.967 \\
\hline Month 3 & 3 & 0.25 & 0.08 & 0.01 & 0.938 \\
\hline Month 4 & 4 & 0.33 & 0.09 & 0.01 & 0.908 \\
\hline Month 5 & 5 & 0.42 & 0.10 & 0.02 & 0.877 \\
\hline Month 6 & 6 & 0.50 & 0.12 & 0.02 & 0.845 \\
\hline Month 7 & 7 & 0.58 & 0.14 & 0.03 & 0.813 \\
\hline Month 8 & 8 & 0.67 & 0.16 & 0.04 & 0.779 \\
\hline Month 9 & 9 & 0.75 & 0.18 & 0.06 & 0.743 \\
\hline Month 10 & 10 & 0.83 & 0.21 & 0.08 & 0.705 \\
\hline Month 11 & 11 & 0.92 & 0.24 & 0.10 & 0.665 \\
\hline Month 12 & 12 & 1.00 & 0.27 & 0.14 & 0.622 \\
\hline Month 13 & 13 & 1.08 & 0.30 & 0.18 & 0.576 \\
\hline Month 14 & 14 & 1.17 & 0.34 & 0.23 & 0.526 \\
\hline Month 15 & 15 & 1.25 & 0.38 & 0.28 & 0.473 \\
\hline Month 16 & 16 & 1.33 & 0.42 & 0.35 & 0.417 \\
\hline Month 17 & 17 & 1.42 & 0.46 & 0.42 & 0.361 \\
\hline Month 18 & 18 & 1.50 & 0.50 & 0.50 & 0.304 \\
\hline Month 19 & 19 & 1.58 & 0.54 & 0.58 & 0.250 \\
\hline Month 20 & 20 & 1.67 & 0.58 & 0.65 & 0.201 \\
\hline Month 21 & 21 & 1.75 & 0.62 & 0.71 & 0.156 \\
\hline \begin{tabular}{|l|} 
Month 22 \\
\end{tabular} & 22 & 1.83 & 0.66 & 0.77 & 0.119 \\
\hline Month 23 & 23 & 1.92 & 0.70 & 0.82 & 0.088 \\
\hline \begin{tabular}{|l|} 
Month 24 \\
\end{tabular} & 24 & 2.00 & 0.73 & 0.86 & 0.064 \\
\hline Month 25 & 25 & 2.08 & 0.76 & 0.90 & 0.046 \\
\hline \begin{tabular}{|l|} 
Month 26 \\
\end{tabular} & 26 & 2.17 & 0.79 & 0.92 & 0.032 \\
\hline \begin{tabular}{|l|} 
Month 27 \\
\end{tabular} & 27 & 2.25 & 0.82 & 0.94 & 0.022 \\
\hline Month 28 & 28 & 2.33 & 0.84 & 0.96 & 0.015 \\
\hline Month 29 & 29 & 2.42 & 0.86 & 0.97 & 0.010 \\
\hline Month 30 & 30 & 2.50 & 0.88 & 0.98 & 0.006 \\
\hline Month 31 & 31 & 2.58 & 0.90 & 0.98 & 0.004 \\
\hline Month 32 & 32 & 2.67 & 0.91 & 0.99 & 0.003 \\
\hline \begin{tabular}{|l|} 
Month 33 \\
\end{tabular} & 33 & 2.75 & 0.92 & 0.99 & 0.002 \\
\hline Month 34 & 34 & 2.83 & 0.93 & 0.99 & 0.001 \\
\hline \begin{tabular}{|l|} 
Month 35 \\
\end{tabular} & 35 & 2.92 & 0.94 & 0.99 & 0.000 \\
\hline Month 36 & 36 & 3.00 & 0.95 & 1.00 & 0.000 \\
\hline \multicolumn{3}{|c|}{ Project End } & 1.00 & 1.00 & 0.000 \\
\hline
\end{tabular}


Journal of Entrepreneurship and Project Management

ISSN 2520-9116 (Online)

Vol. 6, Issue No.2, pp 1 -37, 2021

www.carijournals.org

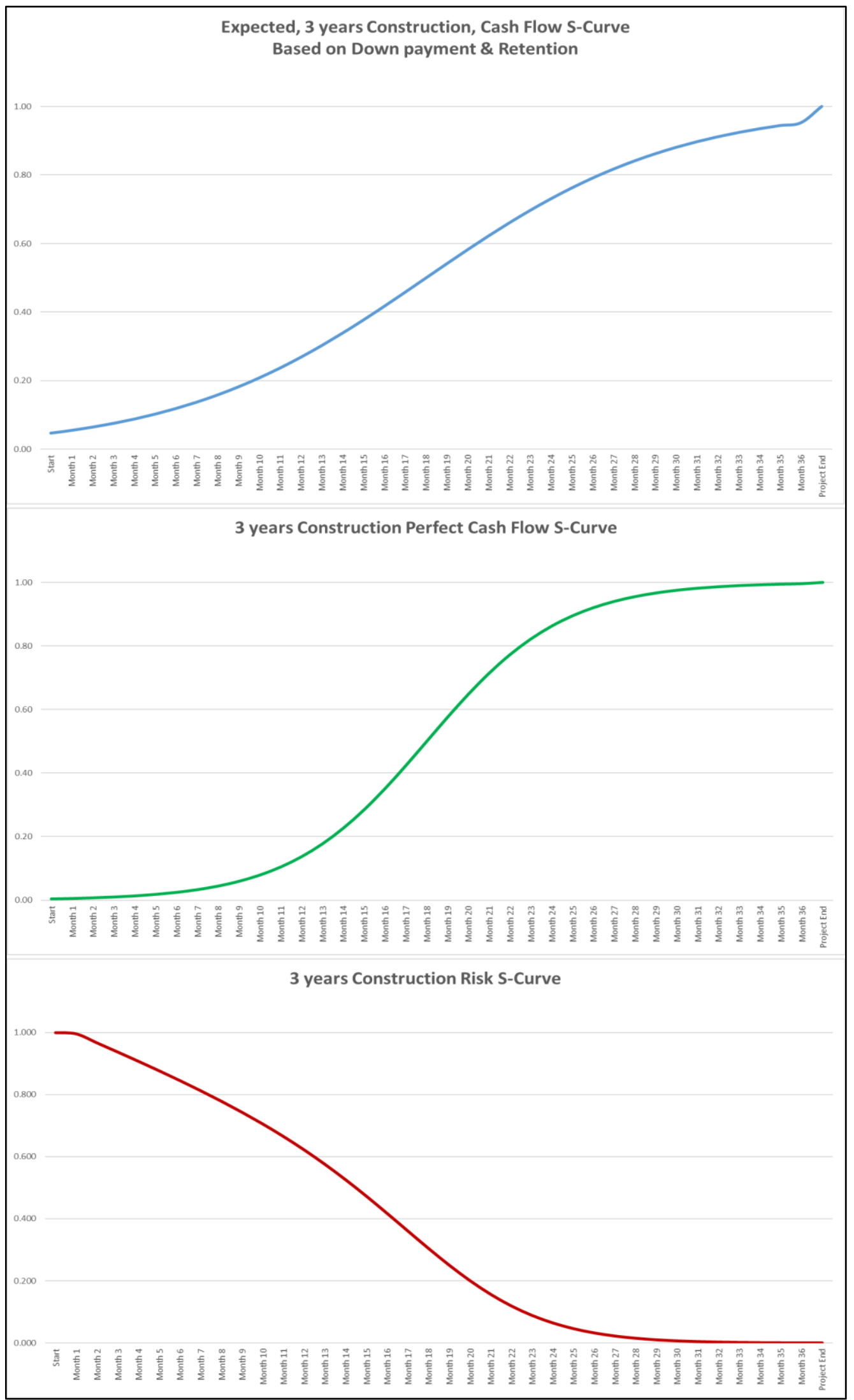

Figure 7. Trending 3 years construction Cash flow and Risk S-Curves based on accepting retention bond. 
Journal of Entrepreneurship and Project Management

ISSN 2520-9116 (Online)

Vol. 6, Issue No.2, pp 1 -37, 2021

www.carijournals.org

Table 7. Identifying 3 years construction Cash flow and Risk S-Curves based on DNP/DLP without retention bond.

\begin{tabular}{|c|c|c|c|c|c|}
\hline \multirow{2}{*}{$\begin{array}{l}\text { Time } \\
\text { Label }\end{array}$} & \multirow{2}{*}{$\begin{array}{l}\text { Month Interval } \\
\text { (n) }\end{array}$} & Year Interval & $\begin{array}{c}\text { Expected Cash Flow S-Curve } \\
\text { Based on Down payment \& } \\
\text { Retention }\end{array}$ & Perfect Cash Flow S-Curve & Risk S-Curve \\
\hline & & $\begin{array}{c}\text { "Month Interval }(\mathrm{n}) " * \\
\text { (1/ "\# of time steps in a } \\
\text { year (i.e., 12)") }\end{array}$ & $\begin{array}{l}\text { 1/(1+(EXP(-2*("Year } \\
\text { Interval"-1.5)))) }\end{array}$ & $\begin{array}{l}\text { 1/(1+(EXP(-3.68*("Year } \\
\text { Interval"-1.5)))) }\end{array}$ & $\begin{array}{c}\text { ((3 -"Year Interval (n-1)")/3)* } \\
\text { (1-"Perfect Cash Flow S-Curve (n-1)") }\end{array}$ \\
\hline Start & $\mathbf{0}$ & 0.00 & 0.05 & 0.00 & 1.000 \\
\hline Month 1 & 1 & 0.08 & 0.06 & 0.01 & 0.996 \\
\hline Month 2 & 2 & 0.17 & 0.06 & 0.01 & 0.967 \\
\hline Month 3 & 3 & 0.25 & 0.08 & 0.01 & 0.938 \\
\hline Month 4 & 4 & 0.33 & 0.09 & 0.01 & 0.908 \\
\hline Month 5 & 5 & 0.42 & 0.10 & 0.02 & 0.877 \\
\hline Month 6 & 6 & 0.50 & 0.12 & 0.02 & 0.845 \\
\hline Month 7 & 7 & 0.58 & 0.14 & 0.03 & 0.813 \\
\hline Month 8 & 8 & 0.67 & 0.16 & 0.04 & 0.779 \\
\hline Month 9 & 9 & 0.75 & 0.18 & 0.06 & 0.743 \\
\hline Month 10 & 10 & 0.83 & 0.21 & 0.08 & 0.705 \\
\hline Month 11 & 11 & 0.92 & 0.24 & 0.10 & 0.665 \\
\hline Month 12 & 12 & 1.00 & 0.27 & 0.14 & 0.622 \\
\hline Month 13 & 13 & 1.08 & 0.30 & 0.18 & 0.576 \\
\hline Month 14 & 14 & 1.17 & 0.34 & 0.23 & 0.526 \\
\hline Month 15 & 15 & 1.25 & 0.38 & 0.28 & 0.473 \\
\hline Month 16 & 16 & 1.33 & 0.42 & 0.35 & 0.417 \\
\hline Month 17 & 17 & 1.42 & 0.46 & 0.42 & 0.361 \\
\hline Month 18 & 18 & 1.50 & 0.50 & 0.50 & 0.304 \\
\hline Month 19 & 19 & 1.58 & 0.54 & 0.58 & 0.250 \\
\hline Month 20 & 20 & 1.67 & 0.58 & 0.65 & 0.201 \\
\hline Month 21 & 21 & 1.75 & 0.62 & 0.71 & 0.156 \\
\hline Month 22 & 22 & 1.83 & 0.66 & 0.77 & 0.119 \\
\hline Month 23 & 23 & 1.92 & 0.70 & 0.82 & 0.088 \\
\hline Month 24 & 24 & 2.00 & 0.73 & 0.86 & 0.064 \\
\hline Month 25 & 25 & 2.08 & 0.76 & 0.90 & 0.046 \\
\hline Month 26 & 26 & 2.17 & 0.79 & 0.92 & 0.032 \\
\hline Month 27 & 27 & 2.25 & 0.82 & 0.94 & 0.022 \\
\hline Month 28 & 28 & 2.33 & 0.84 & 0.96 & 0.015 \\
\hline Month 29 & 29 & 2.42 & 0.86 & 0.97 & 0.010 \\
\hline Month 30 & 30 & 2.50 & 0.88 & 0.98 & 0.006 \\
\hline Month 31 & 31 & 2.58 & 0.90 & 0.98 & 0.004 \\
\hline Month 32 & 32 & 2.67 & 0.91 & 0.99 & 0.003 \\
\hline Month 33 & 33 & 2.75 & 0.92 & 0.99 & 0.002 \\
\hline Month 34 & 34 & 2.83 & 0.93 & 0.99 & 0.001 \\
\hline Month 35 & 35 & 2.92 & 0.94 & 0.99 & 0.000 \\
\hline Month 36 & 36 & 3.00 & 0.95 & 1.00 & 0.000 \\
\hline Month 37 & 37 & 3.08 & 0.95 & 1 & 0.000 \\
\hline Month 38 & 38 & 3.17 & 0.95 & 1 & 0.000 \\
\hline Month 39 & 39 & 3.25 & 0.95 & 1 & 0.000 \\
\hline Month 40 & 40 & 3.33 & 0.95 & 1 & 0.000 \\
\hline Month 41 & 41 & 3.42 & 0.95 & 1 & 0.000 \\
\hline Month 42 & 42 & 3.50 & 0.95 & 1 & 0.000 \\
\hline Month 43 & 43 & 3.58 & 0.95 & 1 & 0.000 \\
\hline Month 44 & 44 & 3.67 & 0.95 & 1 & 0.000 \\
\hline Month 45 & 45 & 3.75 & 0.95 & 1 & 0.000 \\
\hline Month 46 & 46 & 3.83 & 0.95 & 1 & 0.000 \\
\hline Month 47 & 47 & 3.92 & 0.95 & 1 & 0.000 \\
\hline Month 48 & 48 & 4.00 & 0.99 & 1 & 0.000 \\
\hline Month 49 & 49 & 4.08 & 0.99 & 1 & 0.000 \\
\hline Month 50 & 50 & 4.17 & 0.99 & 1 & 0.000 \\
\hline Month 51 & 51 & 4.25 & 0.99 & 1 & 0.000 \\
\hline Month 52 & 52 & 4.33 & 0.99 & 1 & 0.000 \\
\hline Month 53 & 53 & 4.41 & 0.99 & 1 & 0.000 \\
\hline Month 54 & 54 & 4.50 & 0.99 & 1 & 0.000 \\
\hline Month 55 & 55 & 4.58 & 0.99 & 1 & 0.000 \\
\hline Month 56 & 56 & 4.66 & 0.99 & 1 & 0.000 \\
\hline Month 57 & 57 & 4.75 & 0.99 & 1 & 0.000 \\
\hline Month 58 & 58 & 4.83 & 0.99 & 1 & 0.000 \\
\hline Month 59 & 59 & 4.91 & 0.99 & 1 & 0.000 \\
\hline Month 60 & 60 & 5.00 & 0.99 & 1 & 0.000 \\
\hline Month 61 & 61 & 5.08 & 0.99 & 1 & 0.000 \\
\hline Month 62 & 62 & 5.16 & 0.99 & 1 & 0.000 \\
\hline Month 63 & 63 & 5.25 & 0.99 & 1 & 0.000 \\
\hline Month 64 & 64 & 5.33 & 0.99 & 1 & 0.000 \\
\hline Month 65 & 65 & 5.41 & 0.99 & 1 & 0.000 \\
\hline Month 66 & 66 & 5.50 & 0.99 & 1 & 0.000 \\
\hline Month 67 & 67 & 5.58 & 0.99 & 1 & 0.000 \\
\hline Month 68 & 68 & 5.66 & 0.99 & 1 & 0.000 \\
\hline Month 69 & 69 & 5.75 & 0.99 & 1 & 0.000 \\
\hline Month 70 & 70 & 5.83 & 0.99 & 1 & 0.000 \\
\hline Month 71 & 71 & 5.91 & 0.99 & 1 & 0.000 \\
\hline Month 72 & 72 & 6.00 & 1.00 & 1 & 0.000 \\
\hline & Projec & & 1.00 & 1 & 0.000 \\
\hline
\end{tabular}


Journal of Entrepreneurship and Project Management

ISSN 2520-9116 (Online)

Vol. 6, Issue No.2, pp $1-37,2021$

www.carijournals.org

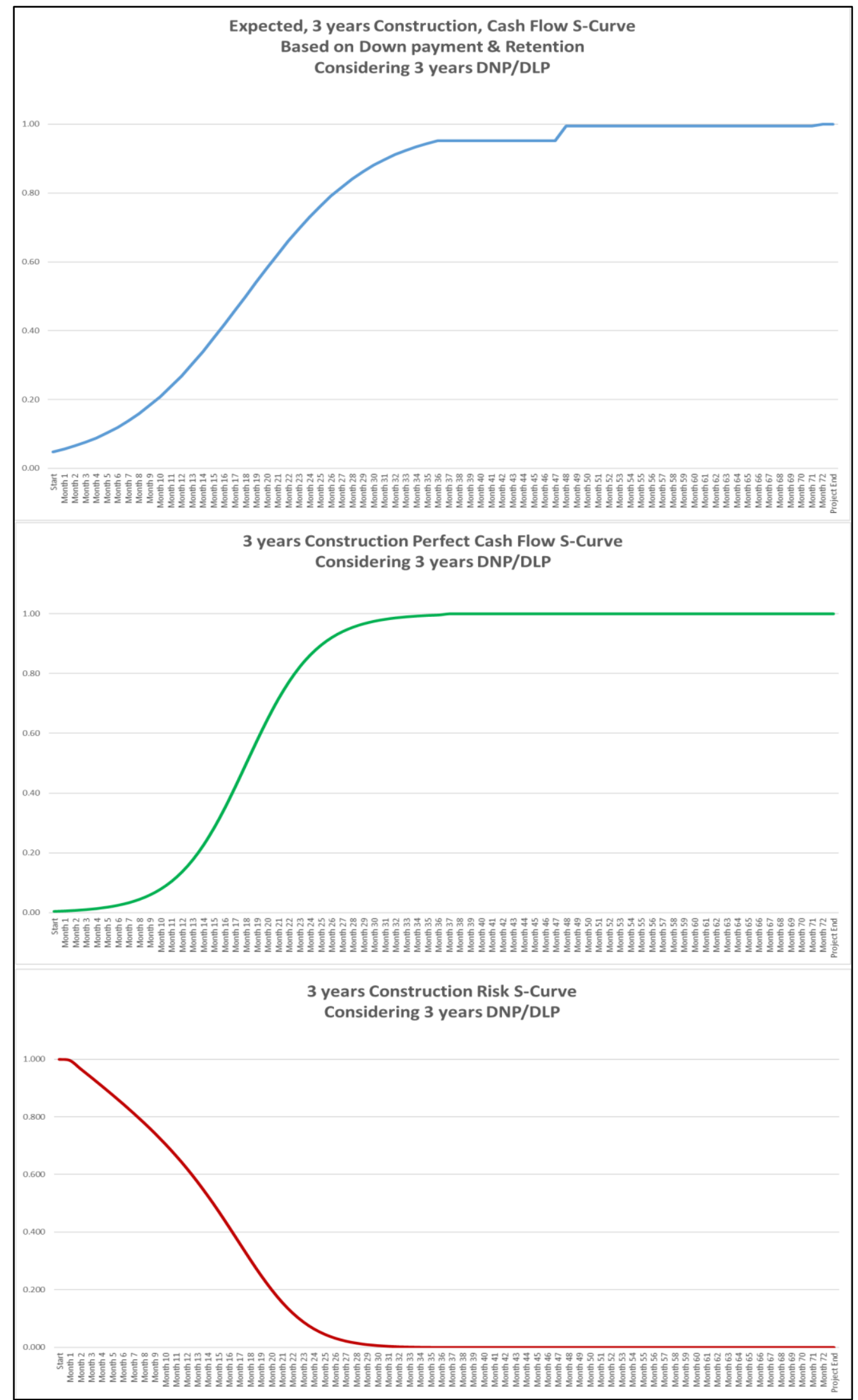

Figure 8. Trending 3 years construction Cash flow and Risk S-Curves based on DNP/DLP without retention bond. 
Journal of Entrepreneurship and Project Management

ISSN 2520-9116 (Online)

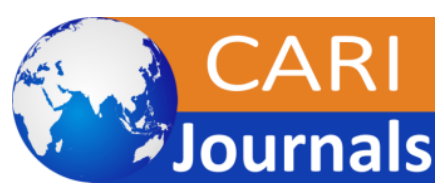

Vol. 6, Issue No.2, pp 1 -37, 2021

www.carijournals.org

Table 8. Identifying 4 years construction Cash flow and Risk S-Curve based on accepting retention bond.

\begin{tabular}{|c|c|c|c|c|c|}
\hline \multirow{2}{*}{$\begin{array}{l}\text { Time } \\
\text { Label }\end{array}$} & \multirow{2}{*}{$\begin{array}{l}\text { Month Interval } \\
\text { (n) }\end{array}$} & Year Interval & $\begin{array}{c}\text { Expected Cash Flow S-Curve } \\
\text { Based on Down payment \& } \\
\text { Retention }\end{array}$ & Perfect Cash Flow S-Curve & Risk S-Curve \\
\hline & & $\begin{array}{c}\text { "Month Interval }(\mathrm{n}) " * \\
\text { (1/ "\# of time steps in } \\
\text { a year (i.e., 12)") }\end{array}$ & $\begin{array}{l}\text { 1/(1+(EXP(-1.46*("Year } \\
\text { Interval"-2)))) }\end{array}$ & $\begin{array}{l}\text { 1/(1+(EXP(-2.65*("Year } \\
\text { Interval"-2)))) }\end{array}$ & $\begin{array}{c}((4 \text {-"Year Interval }(n-1) ") / 4)^{*} \\
(1-" P e r f e c t \text { Cash Flow S-Curve (n-1)") }\end{array}$ \\
\hline Start & 0 & 0.00 & 0.05 & 0.00 & 1.000 \\
\hline Month 1 & 1 & 0.08 & 0.06 & 0.01 & 0.995 \\
\hline Month 2 & 2 & 0.17 & 0.06 & 0.01 & 0.973 \\
\hline Month 3 & 3 & 0.25 & 0.07 & 0.01 & 0.951 \\
\hline Month 4 & 4 & 0.33 & 0.08 & 0.01 & 0.929 \\
\hline Month 5 & 5 & 0.42 & 0.09 & 0.01 & 0.906 \\
\hline Month 6 & 6 & 0.50 & 0.10 & 0.02 & 0.883 \\
\hline Month 7 & 7 & 0.58 & 0.11 & 0.02 & 0.859 \\
\hline Month 8 & 8 & 0.67 & 0.12 & 0.03 & 0.835 \\
\hline Month 9 & 9 & 0.75 & 0.14 & 0.04 & 0.810 \\
\hline Month 10 & 10 & 0.83 & 0.15 & 0.04 & 0.784 \\
\hline Month 11 & 11 & 0.92 & 0.17 & 0.05 & 0.757 \\
\hline Month 12 & 12 & 1.00 & 0.19 & 0.07 & 0.730 \\
\hline Month 13 & 13 & 1.08 & 0.21 & 0.08 & 0.701 \\
\hline Month 14 & 14 & 1.17 & 0.23 & 0.10 & 0.670 \\
\hline Month 15 & 15 & 1.25 & 0.25 & 0.12 & 0.638 \\
\hline Month 16 & 16 & 1.33 & 0.27 & 0.15 & 0.605 \\
\hline Month 17 & 17 & 1.42 & 0.30 & 0.18 & 0.570 \\
\hline Month 18 & 18 & 1.50 & 0.33 & 0.21 & 0.533 \\
\hline Month 19 & 19 & 1.58 & 0.35 & 0.25 & 0.494 \\
\hline Month 20 & 20 & 1.67 & 0.38 & 0.29 & 0.454 \\
\hline Month 21 & 21 & 1.75 & 0.41 & 0.34 & 0.413 \\
\hline Month 22 & 22 & 1.83 & 0.44 & 0.39 & 0.371 \\
\hline Month 23 & 23 & 1.92 & 0.47 & 0.44 & 0.330 \\
\hline Month 24 & 24 & 2.00 & 0.50 & 0.50 & 0.289 \\
\hline Month 25 & 25 & 2.08 & 0.53 & 0.55 & 0.250 \\
\hline Month 26 & 26 & 2.17 & 0.56 & 0.61 & 0.214 \\
\hline Month 27 & 27 & 2.25 & 0.59 & 0.66 & 0.180 \\
\hline Month 28 & 28 & 2.33 & 0.62 & 0.71 & 0.149 \\
\hline Month 29 & 29 & 2.42 & 0.65 & 0.75 & 0.122 \\
\hline Month 30 & 30 & 2.50 & 0.67 & 0.79 & 0.099 \\
\hline Month 31 & 31 & 2.58 & 0.70 & 0.82 & 0.079 \\
\hline Month 32 & 32 & 2.67 & 0.73 & 0.85 & 0.062 \\
\hline Month 33 & 33 & 2.75 & 0.75 & 0.88 & 0.049 \\
\hline Month 34 & 34 & 2.83 & 0.77 & 0.90 & 0.038 \\
\hline Month 35 & 35 & 2.92 & 0.79 & 0.92 & 0.029 \\
\hline Month 36 & 36 & 3.00 & 0.81 & 0.93 & 0.022 \\
\hline Month 37 & 37 & 3.08 & 0.83 & 0.95 & 0.017 \\
\hline Month 38 & 38 & 3.17 & 0.85 & 0.96 & 0.012 \\
\hline Month 39 & 39 & 3.25 & 0.86 & 0.96 & 0.009 \\
\hline Month 40 & 40 & 3.33 & 0.87 & 0.97 & 0.007 \\
\hline Month 41 & 41 & 3.42 & 0.89 & 0.98 & 0.005 \\
\hline Month 42 & 42 & 3.50 & 0.90 & 0.98 & 0.003 \\
\hline Month 43 & 43 & 3.58 & 0.91 & 0.99 & 0.002 \\
\hline Month 44 & 44 & 3.67 & 0.92 & 0.99 & 0.002 \\
\hline Month 45 & 45 & 3.75 & 0.93 & 0.99 & 0.001 \\
\hline Month 46 & 46 & 3.83 & 0.94 & 0.99 & 0.001 \\
\hline Month 47 & 47 & 3.92 & 0.94 & 0.99 & 0.000 \\
\hline Month 48 & 48 & 4.00 & 0.95 & 1.00 & 0.000 \\
\hline & Project & End & 1.00 & 1.00 & 0.000 \\
\hline
\end{tabular}


Journal of Entrepreneurship and Project Management

ISSN 2520-9116 (Online)

Vol. 6, Issue No.2, pp 1 -37, 2021

Expected, 4 years Construction, Cash Flow S-Curve

Based on Down payment \& Retention

1.00

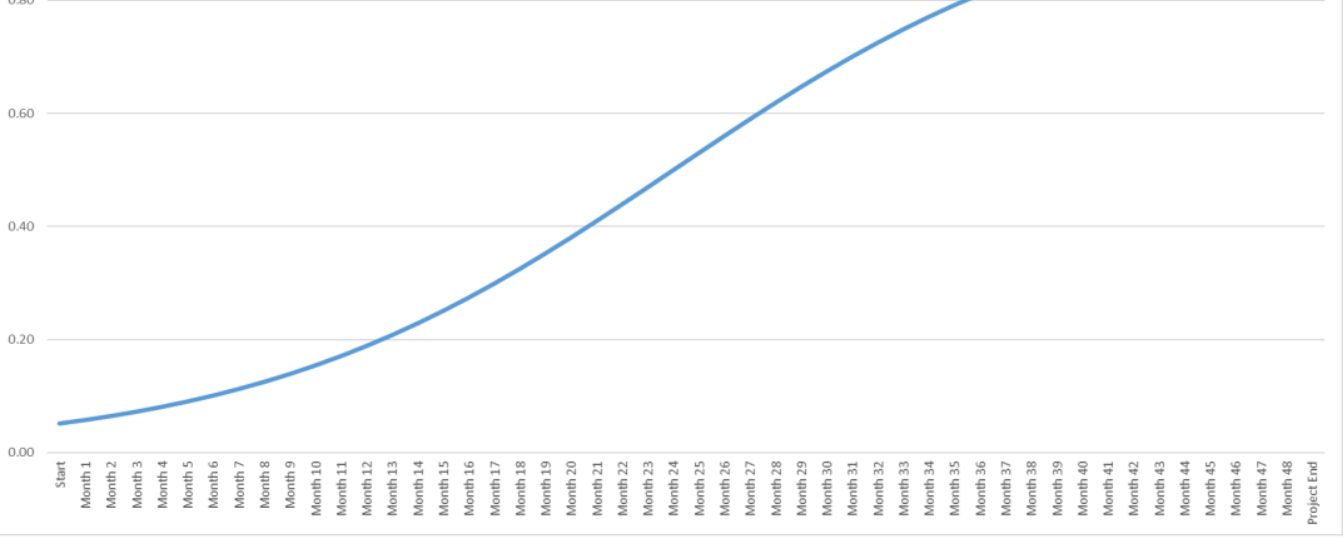

4 years Construction Perfect Cash Flow S-Curve

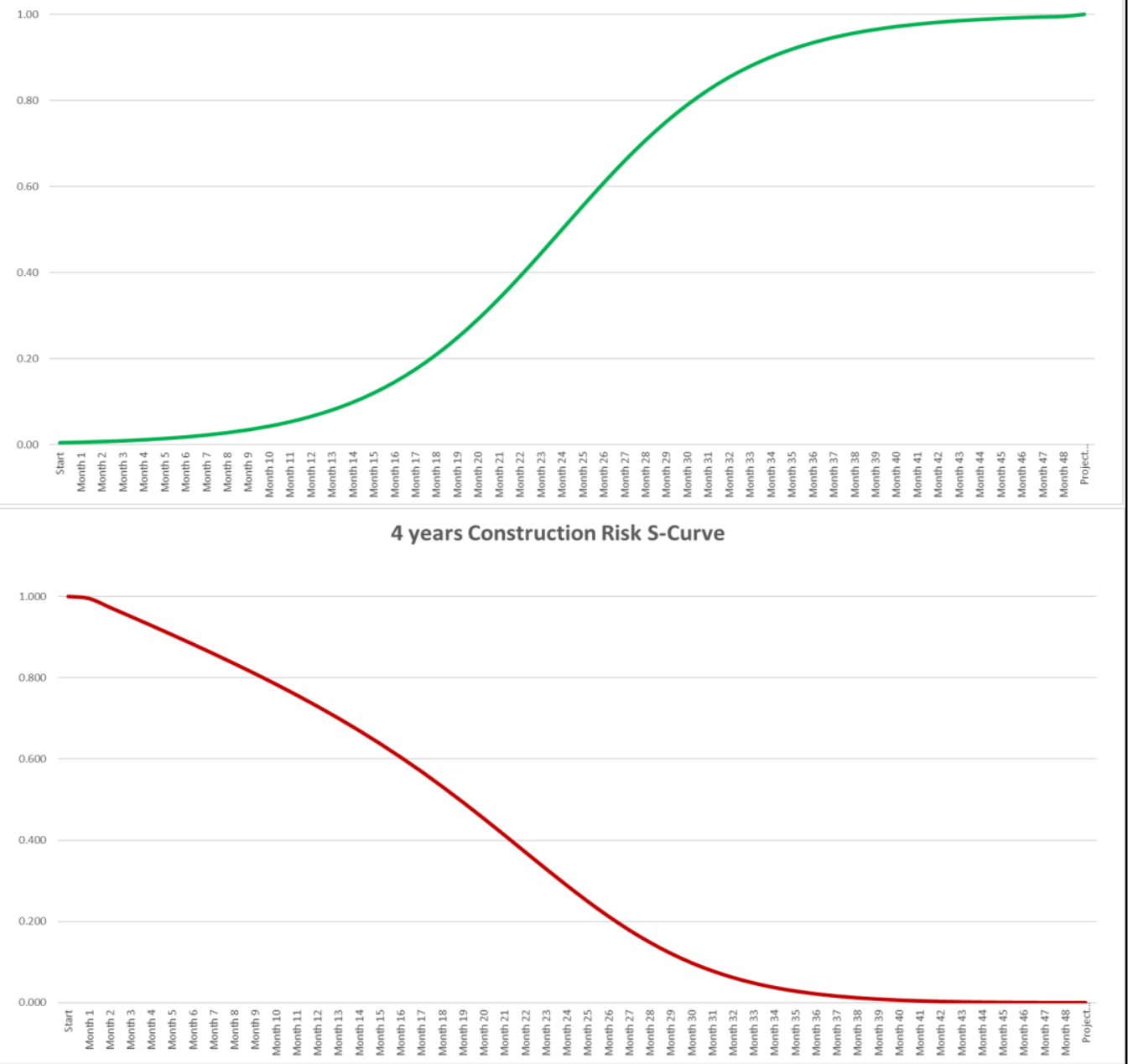

Figure 9. Trending 4 years construction Cash flow and Risk S-Curves based on accepting retention bond. 
Journal of Entrepreneurship and Project Management

ISSN 2520-9116 (Online)

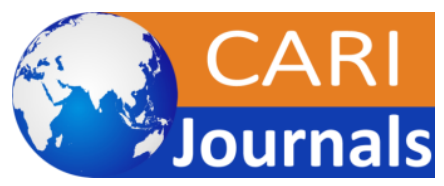

Vol. 6, Issue No.2, pp 1 -37, 2021

www.carijournals.org

Table 9. Identifying 4 years construction Cash flow and Risk S-Curves based on DNP/DLP without retention bond.

\begin{tabular}{|c|c|c|c|c|c|}
\hline \multirow{2}{*}{$\begin{array}{l}\text { Time } \\
\text { Label }\end{array}$} & \multirow{2}{*}{$\begin{array}{l}\text { Month Interval } \\
\text { (n) }\end{array}$} & Year Interval & $\begin{array}{c}\text { Expected Cash Flow S-Curve } \\
\text { Based on Down payment \& } \\
\text { Retention }\end{array}$ & Perfect Cash Flow S-Curve & Risk S-Curve \\
\hline & & \begin{tabular}{|c|} 
"Month Interval $(n) " ~ *$ \\
(1/ "\# of time steps in a \\
year (i.e., 12)")
\end{tabular} & $\begin{array}{l}\text { 1/(1+(EXP(-1.46*("Year } \\
\text { Interval"-2)))) }\end{array}$ & $\begin{array}{l}\text { 1/(1+(EXP(-2.65*("Year } \\
\text { Interval"-2)))) }\end{array}$ & $\begin{array}{c}((4-" \text {-"Year Interval }(n-1) ") / 4)^{*} \\
\text { (1-"Perfect Cash Flow S-Curve (n-1)") }\end{array}$ \\
\hline Start & 0 & \begin{tabular}{|c|}
0.00 \\
\end{tabular} & 0.05 & 0.00 & 1.000 \\
\hline Month 1 & 1 & 0.08 & 0.06 & 0.01 & 0.995 \\
\hline Month 2 & 2 & 0.17 & 0.06 & 0.01 & 0.973 \\
\hline Month 3 & 3 & 0.25 & 0.07 & 0.01 & 0.951 \\
\hline Month 4 & 4 & 0.33 & 0.08 & 0.01 & 0.929 \\
\hline Month 5 & 5 & 0.42 & 0.09 & 0.01 & 0.906 \\
\hline Month 6 & 6 & 0.50 & 0.10 & 0.02 & 0.883 \\
\hline Month 7 & 7 & 0.58 & 0.11 & 0.02 & 0.859 \\
\hline Month 8 & 8 & 0.67 & 0.12 & 0.03 & 0.835 \\
\hline Month 9 & 9 & 0.75 & 0.14 & 0.04 & 0.810 \\
\hline Month 10 & 10 & 0.83 & 0.15 & 0.04 & 0.784 \\
\hline Month 11 & 11 & 0.92 & 0.17 & 0.05 & 0.757 \\
\hline Month 12 & 12 & 1.00 & 0.19 & 0.07 & 0.730 \\
\hline Month 13 & 13 & 1.08 & 0.21 & 0.08 & 0.701 \\
\hline Month 14 & 14 & 1.17 & 0.23 & 0.10 & 0.670 \\
\hline Month 15 & 15 & 1.25 & 0.25 & 0.12 & 0.638 \\
\hline Month 16 & 16 & 1.33 & 0.27 & 0.15 & 0.605 \\
\hline Month 17 & 17 & 1.42 & 0.30 & 0.18 & 0.570 \\
\hline Month 18 & 18 & 1.50 & 0.33 & 0.21 & 0.533 \\
\hline Month 19 & 19 & 1.58 & 0.35 & 0.25 & 0.494 \\
\hline Month 20 & 20 & 1.67 & 0.38 & 0.29 & 0.454 \\
\hline Month 21 & 21 & 1.75 & 0.41 & 0.34 & 0.413 \\
\hline Month 22 & 22 & 1.83 & 0.44 & 0.39 & 0.371 \\
\hline Month 23 & 23 & 1.92 & 0.47 & 0.44 & 0.330 \\
\hline Month 24 & 24 & 2.00 & 0.50 & 0.50 & 0.289 \\
\hline Month 25 & 25 & 2.08 & 0.53 & 0.55 & 0.250 \\
\hline Month 26 & 26 & 2.17 & 0.56 & 0.61 & 0.214 \\
\hline Month 27 & 27 & 2.25 & 0.59 & 0.66 & 0.180 \\
\hline Month 28 & 28 & 2.33 & 0.62 & 0.71 & 0.149 \\
\hline Month 29 & 29 & 2.42 & 0.65 & 0.75 & 0.122 \\
\hline Month 30 & 30 & 2.50 & 0.67 & 0.79 & 0.099 \\
\hline Month 31 & 31 & 2.58 & 0.70 & 0.82 & 0.079 \\
\hline Month 32 & 32 & 2.67 & 0.73 & 0.85 & 0.062 \\
\hline Month 33 & 33 & 2.75 & 0.75 & 0.88 & 0.049 \\
\hline Month 34 & 34 & 2.83 & 0.77 & 0.90 & 0.038 \\
\hline Month 35 & 35 & 2.92 & 0.79 & 0.92 & 0.029 \\
\hline Month 36 & 36 & 3.00 & 0.81 & 0.93 & 0.022 \\
\hline Month 37 & 37 & 3.08 & 0.83 & 0.95 & 0.017 \\
\hline Month 38 & 38 & 3.17 & 0.85 & 0.96 & 0.012 \\
\hline Month 39 & 39 & 3.25 & 0.86 & 0.96 & 0.009 \\
\hline Month 40 & 40 & 3.33 & 0.87 & 0.97 & 0.007 \\
\hline Month 41 & 41 & 3.42 & 0.89 & 0.98 & 0.005 \\
\hline Month 42 & 42 & 3.50 & 0.90 & 0.98 & 0.003 \\
\hline Month 43 & 43 & 3.58 & 0.91 & 0.99 & 0.002 \\
\hline Month 44 & 44 & 3.67 & 0.92 & 0.99 & 0.002 \\
\hline Month 45 & 45 & 3.75 & 0.93 & 0.99 & 0.001 \\
\hline Month 46 & 46 & 3.83 & 0.94 & 0.99 & 0.001 \\
\hline Month 47 & 47 & 3.92 & 0.94 & 0.99 & 0.000 \\
\hline Month 48 & 48 & 4.00 & 0.95 & 1.00 & 0.000 \\
\hline Month 49 & 49 & 4.08 & 0.95 & 1 & 0.000 \\
\hline Month 50 & 50 & 4.17 & 0.95 & 1 & 0.000 \\
\hline Month 51 & 51 & 4.25 & 0.95 & 1 & 0.000 \\
\hline Month 52 & 52 & 4.33 & 0.95 & 1 & 0.000 \\
\hline Month 53 & 53 & 4.41 & 0.95 & 1 & 0.000 \\
\hline Month 54 & 54 & 4.50 & 0.95 & 1 & 0.000 \\
\hline Month 55 & 55 & 4.58 & 0.95 & 1 & 0.000 \\
\hline Month 56 & 56 & 4.66 & 0.95 & 1 & 0.000 \\
\hline Month 57 & 57 & 4.75 & 0.95 & 1 & 0.000 \\
\hline Month 58 & 58 & 4.83 & 0.95 & 1 & 0.000 \\
\hline Month 59 & 59 & 4.91 & 0.95 & 1 & 0.000 \\
\hline Month 60 & 60 & 5.00 & 0.99 & 1 & 0.000 \\
\hline Month 61 & 61 & 5.08 & 0.99 & 1 & 0.000 \\
\hline Month 62 & 62 & 5.16 & 0.99 & 1 & 0.000 \\
\hline Month 63 & 63 & 5.25 & 0.99 & 1 & 0.000 \\
\hline Month 64 & 64 & 5.33 & 0.99 & 1 & 0.000 \\
\hline Month 65 & 65 & 5.41 & 0.99 & 1 & 0.000 \\
\hline Month 66 & 66 & 5.50 & 0.99 & 1 & 0.000 \\
\hline Month 67 & 67 & 5.58 & 0.99 & 1 & 0.000 \\
\hline Month 68 & 68 & 5.66 & 0.99 & 1 & 0.000 \\
\hline Month 69 & 69 & 5.75 & 0.99 & 1 & 0.000 \\
\hline Month 70 & 70 & 5.83 & 0.99 & 1 & 0.000 \\
\hline Month 71 & 71 & 5.91 & 0.99 & 1 & 0.000 \\
\hline Month 72 & 72 & 6.00 & 0.99 & 1 & 0.000 \\
\hline Month 73 & 73 & 6.08 & 0.99 & 1 & 0.000 \\
\hline Month 74 & 74 & 6.16 & 0.99 & 1 & 0.000 \\
\hline Month 75 & 75 & 6.25 & 0.99 & 1 & 0.000 \\
\hline Month 76 & 76 & 6.33 & 0.99 & 1 & 0.000 \\
\hline Month 77 & 77 & 6.41 & 0.99 & 1 & 0.000 \\
\hline $\begin{array}{l}\text { Month } 78 \\
\end{array}$ & 78 & 6.50 & 0.99 & 1 & 0.000 \\
\hline Month 79 & 79 & 6.58 & 0.99 & 1 & 0.000 \\
\hline Month 80 & 80 & 6.66 & 0.99 & 1 & 0.000 \\
\hline Month 81 & 81 & 6.75 & 0.99 & 1 & 0.000 \\
\hline Month 82 & 82 & 6.83 & 0.99 & 1 & 0.000 \\
\hline Month 83 & 83 & 6.91 & 0.99 & 1 & 0.000 \\
\hline Month 84 & 84 & 7.00 & 1 & 1 & 0.000 \\
\hline & Project $\mathrm{E}$ & & 1 & 1 & 0.000 \\
\hline
\end{tabular}


Journal of Entrepreneurship and Project Management

ISSN 2520-9116 (Online)

Vol. 6, Issue No.2, pp $1-37,2021$

www.carijournals.org

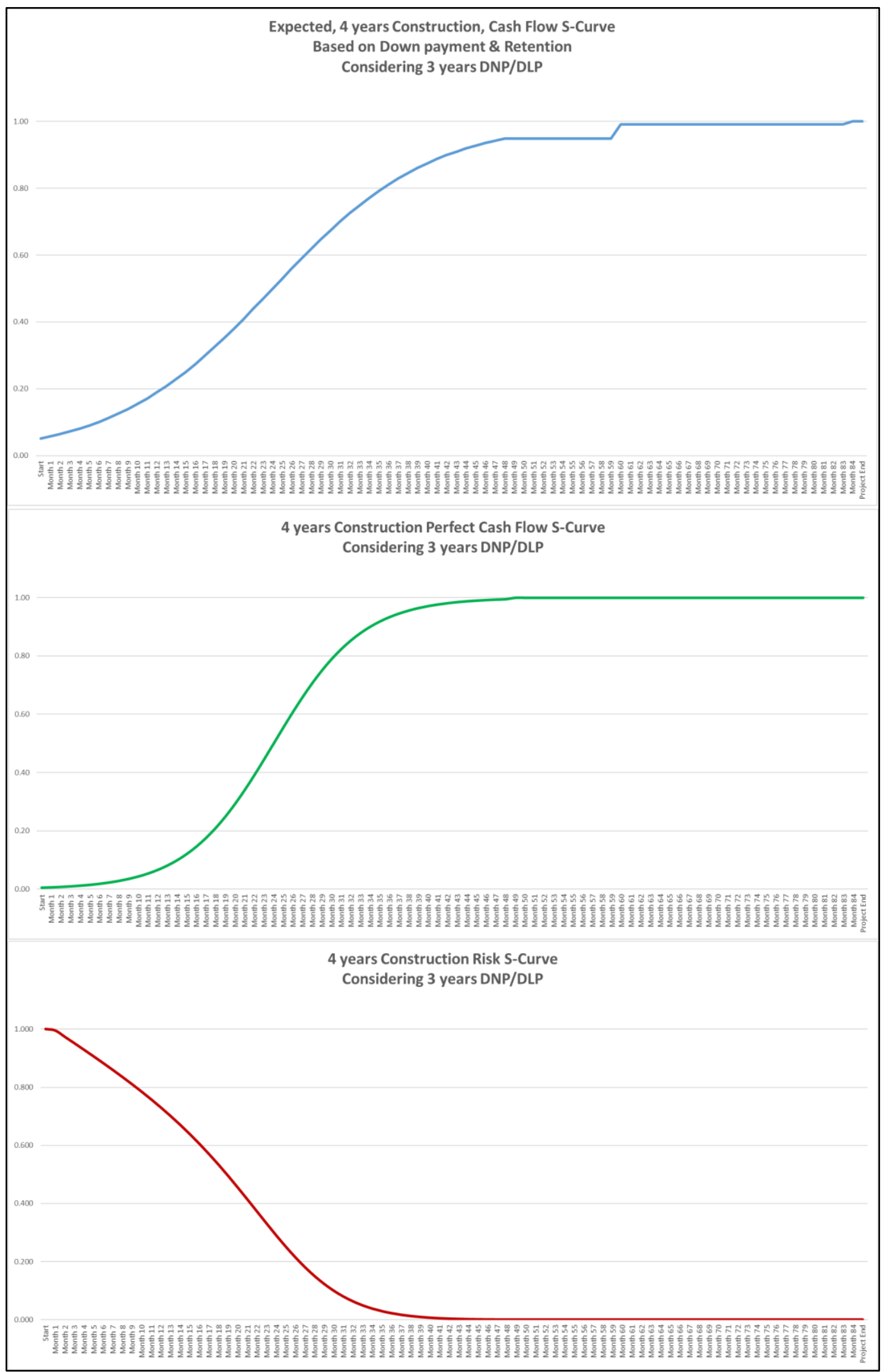

Figure 10. Trending 4 years construction Cash flow and Risk S-Curves based on DNP/DLP without retention bond. 
Journal of Entrepreneurship and Project Management

ISSN 2520-9116 (Online)

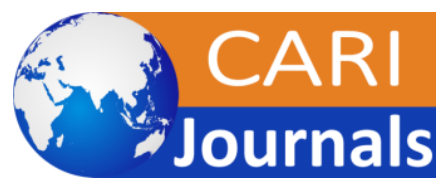

Vol. 6, Issue No.2, pp 1 -37, 2021

WwW.carijournals.org

Table 10. Identifying 5 years construction Cash flow and Risk S-Curves based on accepting retention bond.

\begin{tabular}{|c|c|c|c|c|c|}
\hline \multirow{2}{*}{$\begin{array}{l}\text { Time } \\
\text { Label }\end{array}$} & \multirow{2}{*}{$\begin{array}{l}\text { Month Interval } \\
\text { (n) }\end{array}$} & Year Interval & $\begin{array}{c}\text { Expected Cash Flow S-Curve } \\
\text { Based on Down payment \& } \\
\text { Retention }\end{array}$ & Perfect Cash Flow S-Curve & Risk S-Curve \\
\hline & & \begin{tabular}{|c|} 
Month Interval $(n) " ~ *$ \\
(1/ "\# of time steps in \\
a year (i.e., 12)")
\end{tabular} & $\begin{array}{l}\text { 1/(1+(EXP(-1.2*("Year } \\
\text { Interval"-2.5)))) }\end{array}$ & $\begin{array}{l}\text { 1/(1+(EXP(-2.15*("Year } \\
\text { Interval"-2.5)))) }\end{array}$ & $\begin{array}{c}\text { ((5 -"Year Interval (n-1)")/5)* } \\
\text { (1-"Perfect Cash Flow S-Curve (n-1)") }\end{array}$ \\
\hline Start & 0 & \begin{tabular}{|c|}
0.00 \\
\end{tabular} & 0.05 & 0.00 & 1.000 \\
\hline \begin{tabular}{|l|} 
Month 1 \\
\end{tabular} & 1 & 0.08 & 0.05 & 0.01 & 0.995 \\
\hline Month 2 & 2 & 0.17 & 0.06 & 0.01 & 0.978 \\
\hline Month 3 & 3 & 0.25 & 0.06 & 0.01 & 0.960 \\
\hline Month 4 & 4 & 0.33 & 0.07 & 0.01 & 0.943 \\
\hline Month 5 & 5 & 0.42 & 0.08 & 0.01 & 0.925 \\
\hline Month 6 & 6 & 0.50 & 0.08 & 0.01 & 0.906 \\
\hline Month 7 & 7 & 0.58 & 0.09 & 0.02 & 0.888 \\
\hline Month 8 & 8 & 0.67 & 0.10 & 0.02 & 0.869 \\
\hline Month 9 & 9 & 0.75 & 0.11 & 0.02 & 0.850 \\
\hline Month 10 & 10 & 0.83 & 0.12 & 0.03 & 0.831 \\
\hline Month 11 & 11 & 0.92 & 0.13 & 0.03 & 0.811 \\
\hline Month 12 & 12 & 1.00 & 0.14 & 0.04 & 0.790 \\
\hline Month 13 & 13 & 1.08 & 0.15 & 0.05 & 0.770 \\
\hline Month 14 & 14 & 1.17 & 0.17 & 0.05 & 0.748 \\
\hline Month 15 & 15 & 1.25 & 0.18 & 0.06 & 0.726 \\
\hline Month 16 & 16 & 1.33 & 0.20 & 0.08 & 0.702 \\
\hline Month 17 & 17 & 1.42 & 0.21 & 0.09 & 0.678 \\
\hline Month 18 & 18 & 1.50 & 0.23 & 0.10 & 0.653 \\
\hline Month 19 & 19 & 1.58 & 0.25 & 0.12 & 0.627 \\
\hline Month 20 & 20 & 1.67 & 0.27 & 0.14 & 0.600 \\
\hline Month 21 & 21 & 1.75 & 0.29 & 0.17 & 0.572 \\
\hline \begin{tabular}{|l} 
Month 22 \\
\end{tabular} & 22 & 1.83 & 0.31 & 0.19 & 0.542 \\
\hline Month 23 & 23 & 1.92 & 0.33 & 0.22 & 0.512 \\
\hline Month 24 & 24 & 2.00 & 0.35 & 0.25 & 0.480 \\
\hline \begin{tabular}{|l|} 
Month 25 \\
\end{tabular} & 25 & 2.08 & 0.38 & 0.29 & 0.448 \\
\hline Month 26 & 26 & 2.17 & 0.40 & 0.33 & 0.415 \\
\hline Month 27 & 27 & 2.25 & 0.43 & 0.37 & 0.381 \\
\hline \begin{tabular}{|l|} 
Month 28 \\
\end{tabular} & 28 & 2.33 & 0.45 & 0.41 & 0.348 \\
\hline Month 29 & 29 & 2.42 & 0.47 & 0.45 & 0.314 \\
\hline Month 30 & 30 & 2.50 & 0.50 & 0.50 & 0.282 \\
\hline \begin{tabular}{|l|} 
Month 31 \\
\end{tabular} & 31 & 2.58 & 0.52 & 0.54 & 0.250 \\
\hline Month 32 & 32 & 2.67 & 0.55 & 0.59 & 0.220 \\
\hline Month 33 & 33 & 2.75 & 0.57 & 0.63 & 0.192 \\
\hline Month 34 & 34 & 2.83 & 0.60 & 0.67 & 0.166 \\
\hline \begin{tabular}{|l|} 
Month 35 \\
\end{tabular} & 35 & 2.92 & 0.62 & 0.71 & 0.142 \\
\hline Month 36 & 36 & 3.00 & 0.65 & 0.75 & 0.121 \\
\hline Month 37 & 37 & 3.08 & 0.67 & 0.78 & 0.102 \\
\hline \begin{tabular}{|l} 
Month 38 \\
\end{tabular} & 38 & 3.17 & 0.69 & 0.81 & 0.085 \\
\hline Month 39 & 39 & 3.25 & 0.71 & 0.83 & 0.071 \\
\hline Month 40 & 40 & 3.33 & 0.73 & 0.86 & 0.058 \\
\hline \begin{tabular}{|l|} 
Month 41 \\
\end{tabular} & 41 & 3.42 & 0.75 & 0.88 & 0.048 \\
\hline Month 42 & 42 & 3.50 & 0.77 & 0.90 & 0.039 \\
\hline Month 43 & 43 & 3.58 & 0.79 & 0.91 & 0.031 \\
\hline \begin{tabular}{|l|} 
Month 44 \\
\end{tabular} & 44 & 3.67 & 0.80 & 0.92 & 0.025 \\
\hline \begin{tabular}{|l|} 
Month 45 \\
\end{tabular} & 45 & 3.75 & 0.82 & 0.94 & 0.020 \\
\hline Month 46 & 46 & 3.83 & 0.83 & 0.95 & 0.016 \\
\hline Month 47 & 47 & 3.92 & 0.85 & 0.95 & 0.013 \\
\hline \begin{tabular}{|l|} 
Month 48 \\
\end{tabular} & 48 & 4.00 & 0.86 & 0.96 & 0.010 \\
\hline Month 49 & 49 & 4.08 & 0.87 & 0.97 & 0.008 \\
\hline Month 50 & 50 & 4.17 & 0.88 & 0.97 & 0.006 \\
\hline \begin{tabular}{|l|} 
Month 51 \\
\end{tabular} & 51 & 4.25 & 0.89 & 0.98 & 0.005 \\
\hline Month 52 & 52 & 4.33 & 0.90 & 0.98 & 0.003 \\
\hline Month 53 & 53 & 4.41 & 0.91 & 0.98 & 0.003 \\
\hline Month 54 & 54 & 4.50 & 0.92 & 0.99 & 0.002 \\
\hline Month 55 & 55 & 4.58 & 0.92 & 0.99 & 0.001 \\
\hline Month 56 & 56 & 4.66 & 0.93 & 0.99 & 0.001 \\
\hline \begin{tabular}{|l} 
Month 57 \\
\end{tabular} & 57 & 4.75 & 0.94 & 0.99 & 0.001 \\
\hline \begin{tabular}{|l|} 
Month 58 \\
\end{tabular} & 58 & 4.83 & 0.94 & 0.99 & 0.000 \\
\hline Month 59 & 59 & 4.91 & 0.95 & 0.99 & 0.000 \\
\hline Month 60 & 60 & 5.00 & 0.95 & 1.00 & 0.000 \\
\hline & Project & End & 1.00 & 1.00 & 0.000 \\
\hline
\end{tabular}


Journal of Entrepreneurship and Project Management

ISSN 2520-9116 (Online)

Vol. 6, Issue No.2, pp 1 -37, 2021

www.carijournals.org

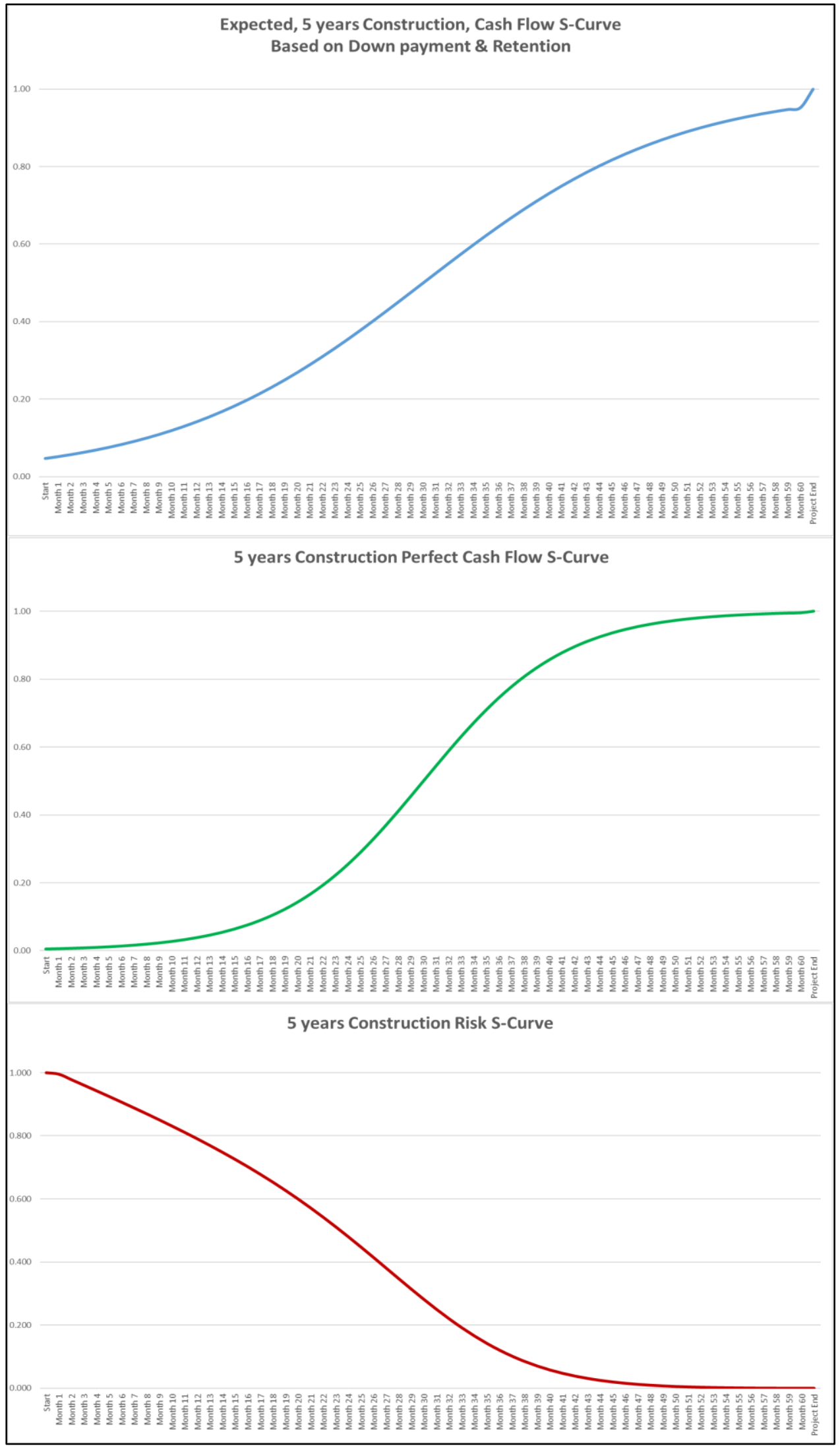

Figure 11. Trending 5 years construction Cash flow and Risk S-Curves based on accepting retention bond. 
Journal of Entrepreneurship and Project Management

ISSN 2520-9116 (Online)

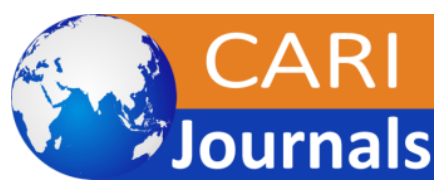

Vol. 6, Issue No.2, pp 1 -37, 2021

www.carijournals.org

Table 11. Identifying 5 years construction Cash flow and Risk $S$-Curves based on DNP/DLP without retention bond.

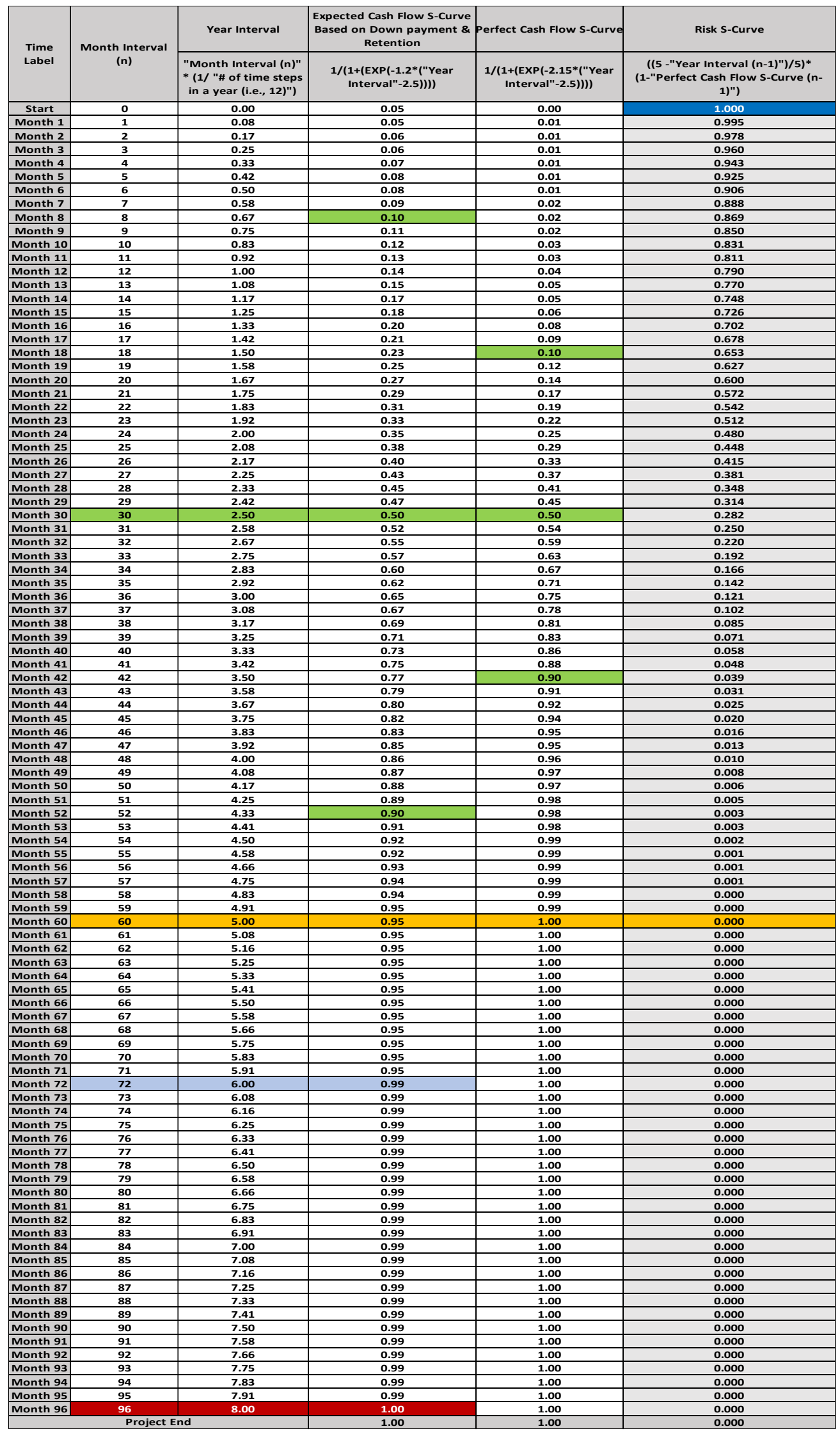


Journal of Entrepreneurship and Project Management

ISSN 2520-9116 (Online)

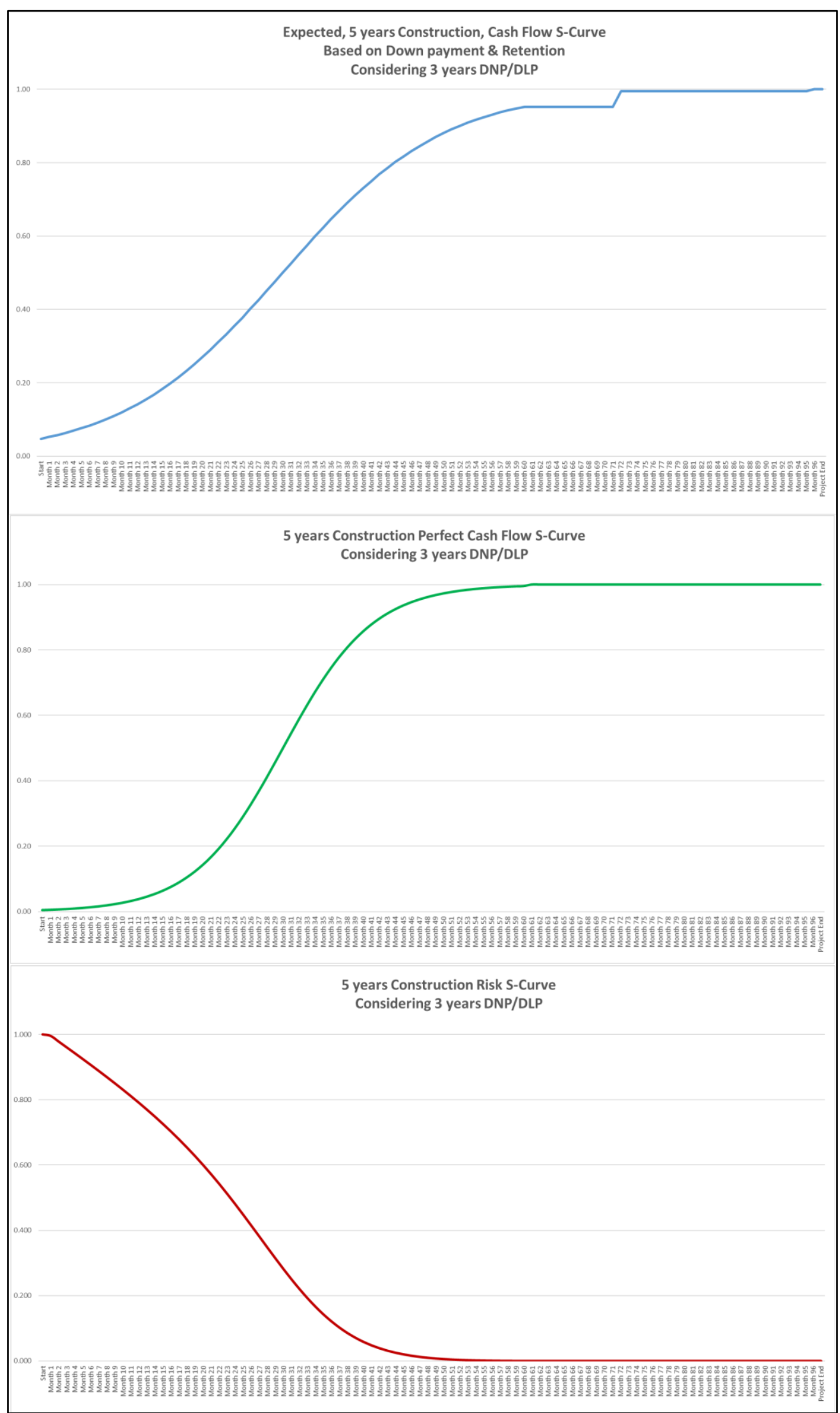

Figure 12. Trending 5 years construction Cash flow and Risk S-Curves based on DNP/DLP without retention bond. 


\subsection{Cash Flow Area Method Approaches}

According to previous research, construction projects' cash flow estimation studies approach area method analysis, as shown in Figure 3 (Konior \& Szóstak 2020). This approach is achieved by plotting previous similar projects S-Curves and create an area that includes all potted points (Konior \& Szóstak 2020). It is then merged with the estimated cash flow to create an updated area, including actual and estimated cash flows (Konior \& Szóstak 2020). This area is used to identify construction projects' cash flow S-Curve range in each time step (Cristóbal 2017; Wang et al. 2016; Odeyinka, Lowe \& Kaka 2013; Konior \& Szóstak 2020). Researchers stated that there are other methods and models for cash flow estimation such as mathematical equations, building information modelling, and baseline forecast (Cristóbal 2017; Wang et al. 2016; Odeyinka, Lowe \& Kaka 2013; Konior \& Szóstak 2020). They are too complicated and do not help plan or support construction project management (Konior \& Szóstak 2020).

In this part of the article, cash flow tolerance possibility estimation based on the chosen verification projects 1, 2, and 3 are analysed as per summary tables 12 to 15 . The area analysis is based on 3 actual projects cash flow and the developed cash flow s-curve. Therefore, this research study approaches the cash flows-curve area method to provide "the easy-to-apply" cash flow model (Konior \& Szóstak 2020, p. 19). This will support planning and managing the project (i.e., monitoring and control (Konior \& Szóstak 2020). It is proved that this approach is a helpful tool (i.e., model) for both investors (i.e., clients) and contractors (Konior \& Szóstak 2020). The following equations 10 to 16 are used to create tables 12 to 15.

$$
\begin{aligned}
& \text { X3 }=\text { X1-X2 } \\
& \text { X4 }=\text { Absolute }(X 3+X 1) \\
& \text { X5 }=\text { Absolute }(X 3-X 1) \\
& \text { X6 }=\text { X4-X5 } \\
& \text { X7 }=\text { MAX X4 value of all selected real projects } \\
& \text { X8 }=\text { MIN X5 value of all selected real projects } \\
& \text { X9 }=\text { X7-X8 }
\end{aligned}
$$

$\mathrm{X} 1$ is the Estimated Cash Flow simple s-curve.

$\mathrm{X} 2$ is the Actual Project Paid Cash Flow for Housing Project.

$\mathrm{X} 3$ is the Deference Between Actual and Estimated Cash Flow.

$\mathrm{X} 4$ is the Project's MAX S-Curve Absolute Error.

X5 is the Project's MIN S-Curve Absolute Error.

X6 is the Project's Error of Each Time Step Cash Flow.

$\mathrm{X} 7$ is the Combined MAX S-Curve Absolute Error.

$\mathrm{X} 8$ is the Combined MIN S-Curve Absolute Error.

X9 is the Total Cash Flow Error (Time Step Cash Flow Fluctuation Possibility). 
Table 12. It is showing verification projects used for this research area method and how much cost cash flow can fluctuate each time step including project 1 .

\begin{tabular}{|c|c|c|c|c|c|c|}
\hline \multicolumn{7}{|c|}{ Housing Project 1} \\
\hline Years & X1 & $\mathbf{X} 2$ & $\mathbf{X 3}$ & $\mathbf{X} 4$ & X5 & X6 \\
\hline 0 & $2.24 \%$ & $0.00 \%$ & $2.24 \%$ & $4.47 \%$ & $0.00 \%$ & $4.47 \%$ \\
\hline 0.125 & $3.11 \%$ & $3.51 \%$ & $-0.40 \%$ & $3.51 \%$ & $2.70 \%$ & $0.81 \%$ \\
\hline 0.25 & $4.30 \%$ & $5.72 \%$ & $-1.42 \%$ & $5.72 \%$ & $2.88 \%$ & $2.84 \%$ \\
\hline 0.375 & $5.92 \%$ & $8.21 \%$ & $-2.29 \%$ & $8.21 \%$ & $3.64 \%$ & $4.57 \%$ \\
\hline 0.5 & $8.11 \%$ & $10.28 \%$ & $-2.17 \%$ & $10.28 \%$ & $5.94 \%$ & $4.34 \%$ \\
\hline 0.625 & $11.01 \%$ & $12.79 \%$ & $-1.78 \%$ & $12.79 \%$ & $9.22 \%$ & $3.57 \%$ \\
\hline 0.75 & $14.77 \%$ & $16.58 \%$ & $-1.81 \%$ & $16.58 \%$ & $12.96 \%$ & $3.62 \%$ \\
\hline 0.875 & $19.55 \%$ & $18.43 \%$ & $1.12 \%$ & $20.66 \%$ & $18.43 \%$ & $2.23 \%$ \\
\hline 1 & $25.40 \%$ & $19.35 \%$ & $6.05 \%$ & $31.45 \%$ & $19.35 \%$ & $12.10 \%$ \\
\hline 1.125 & $32.31 \%$ & $20.76 \%$ & $11.55 \%$ & $43.86 \%$ & $20.76 \%$ & $23.10 \%$ \\
\hline 1.25 & $40.09 \%$ & $35.39 \%$ & $4.70 \%$ & $44.80 \%$ & $35.39 \%$ & $9.41 \%$ \\
\hline 1.375 & $48.41 \%$ & $42.52 \%$ & $5.89 \%$ & $54.30 \%$ & $42.52 \%$ & $11.78 \%$ \\
\hline 1.5 & $56.82 \%$ & $55.25 \%$ & $1.57 \%$ & $58.40 \%$ & $55.25 \%$ & $3.15 \%$ \\
\hline 1.625 & $64.87 \%$ & $75.56 \%$ & $-10.69 \%$ & $75.56 \%$ & $54.18 \%$ & $21.38 \%$ \\
\hline 1.75 & $72.16 \%$ & $82.72 \%$ & $-10.56 \%$ & $82.72 \%$ & $61.59 \%$ & $21.13 \%$ \\
\hline 1.875 & $78.45 \%$ & $84.49 \%$ & $-6.04 \%$ & $84.49 \%$ & $72.40 \%$ & $12.09 \%$ \\
\hline 2 & $83.65 \%$ & $89.76 \%$ & $-6.11 \%$ & $89.76 \%$ & $77.54 \%$ & $12.22 \%$ \\
\hline 2.125 & $87.80 \%$ & $90.75 \%$ & $-2.95 \%$ & $90.75 \%$ & $84.86 \%$ & $5.89 \%$ \\
\hline 2.25 & $91.03 \%$ & $91.44 \%$ & $-0.41 \%$ & $91.44 \%$ & $90.62 \%$ & $0.82 \%$ \\
\hline 2.375 & $93.48 \%$ & $92.73 \%$ & $0.75 \%$ & $94.24 \%$ & $92.73 \%$ & $1.51 \%$ \\
\hline 2.5 & $95.31 \%$ & $93.72 \%$ & $1.59 \%$ & $96.91 \%$ & $93.72 \%$ & $3.19 \%$ \\
\hline 2.625 & $96.67 \%$ & $94.34 \%$ & $2.33 \%$ & $98.99 \%$ & $94.34 \%$ & $4.65 \%$ \\
\hline 2.75 & $97.65 \%$ & $95.70 \%$ & $1.95 \%$ & $99.61 \%$ & $95.70 \%$ & $3.91 \%$ \\
\hline 2.875 & $98.37 \%$ & $97.72 \%$ & $0.65 \%$ & $99.02 \%$ & $97.72 \%$ & $1.30 \%$ \\
\hline 3 & $100.20 \%$ & $100.00 \%$ & $0.20 \%$ & $100.41 \%$ & $100.00 \%$ & $0.41 \%$ \\
\hline
\end{tabular}

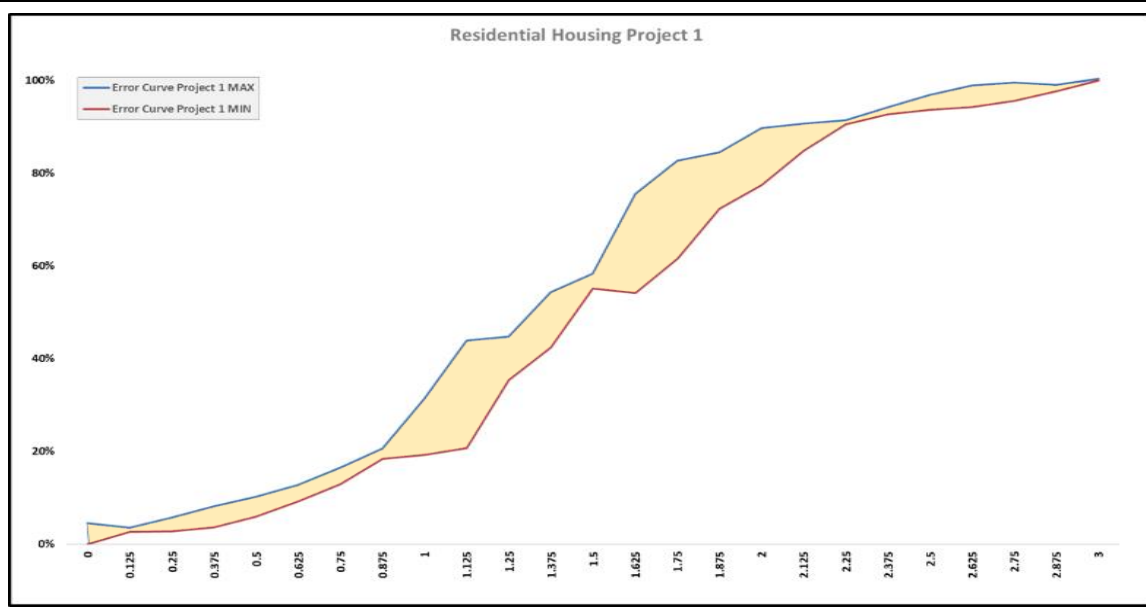

Figure 13. Cash flow area method range of verification project 1 (i.e., within the highlighted area). 
Table 13. It is showing verification projects used for this research area method and how much cost cash flow can fluctuate each time step including project 2.

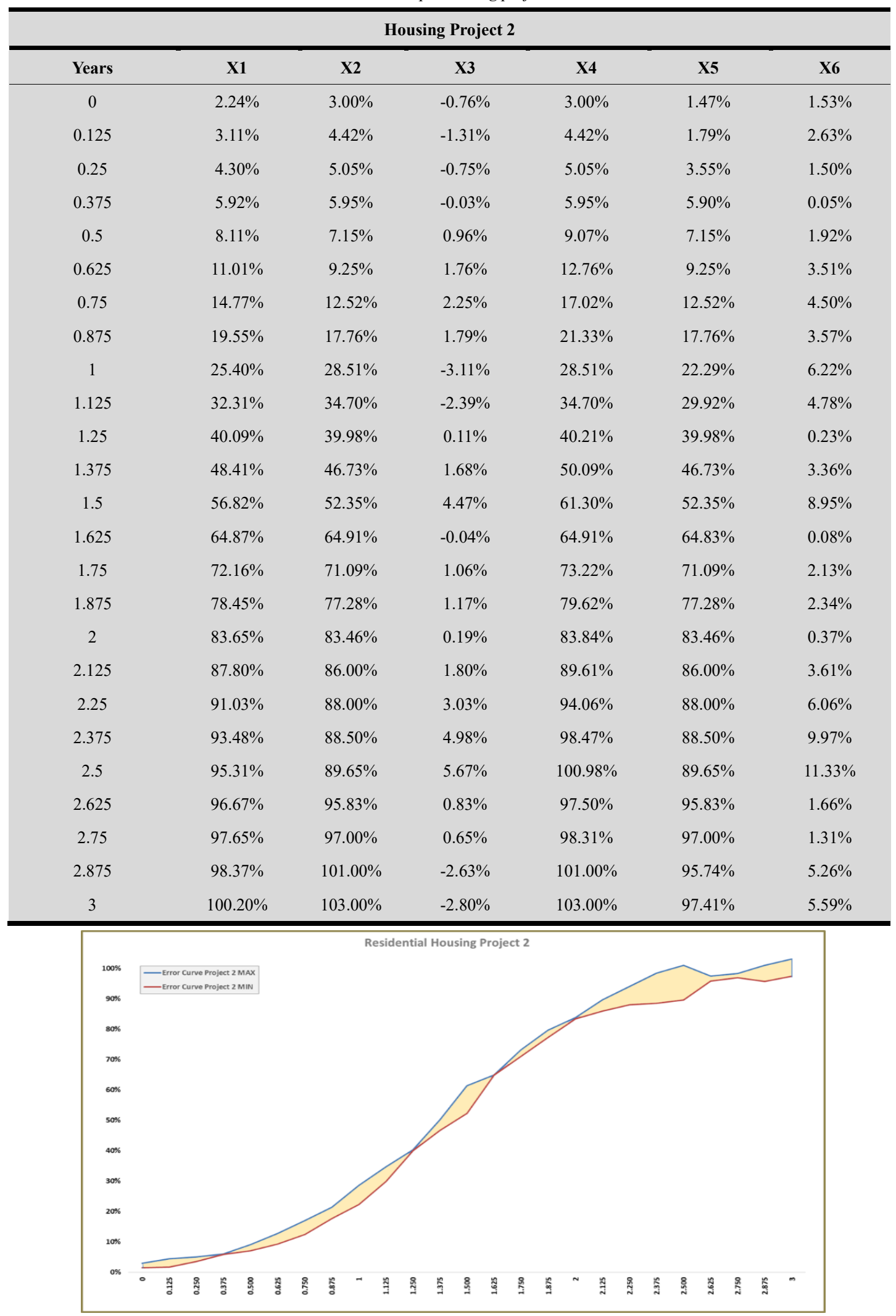

Figure 14. Cash flow area method range of verification project 2 (i.e., within the highlighted area). 
Table 14. It is showing verification projects used for this research area method and how much cost cash flow can fluctuate each time step including project 3.

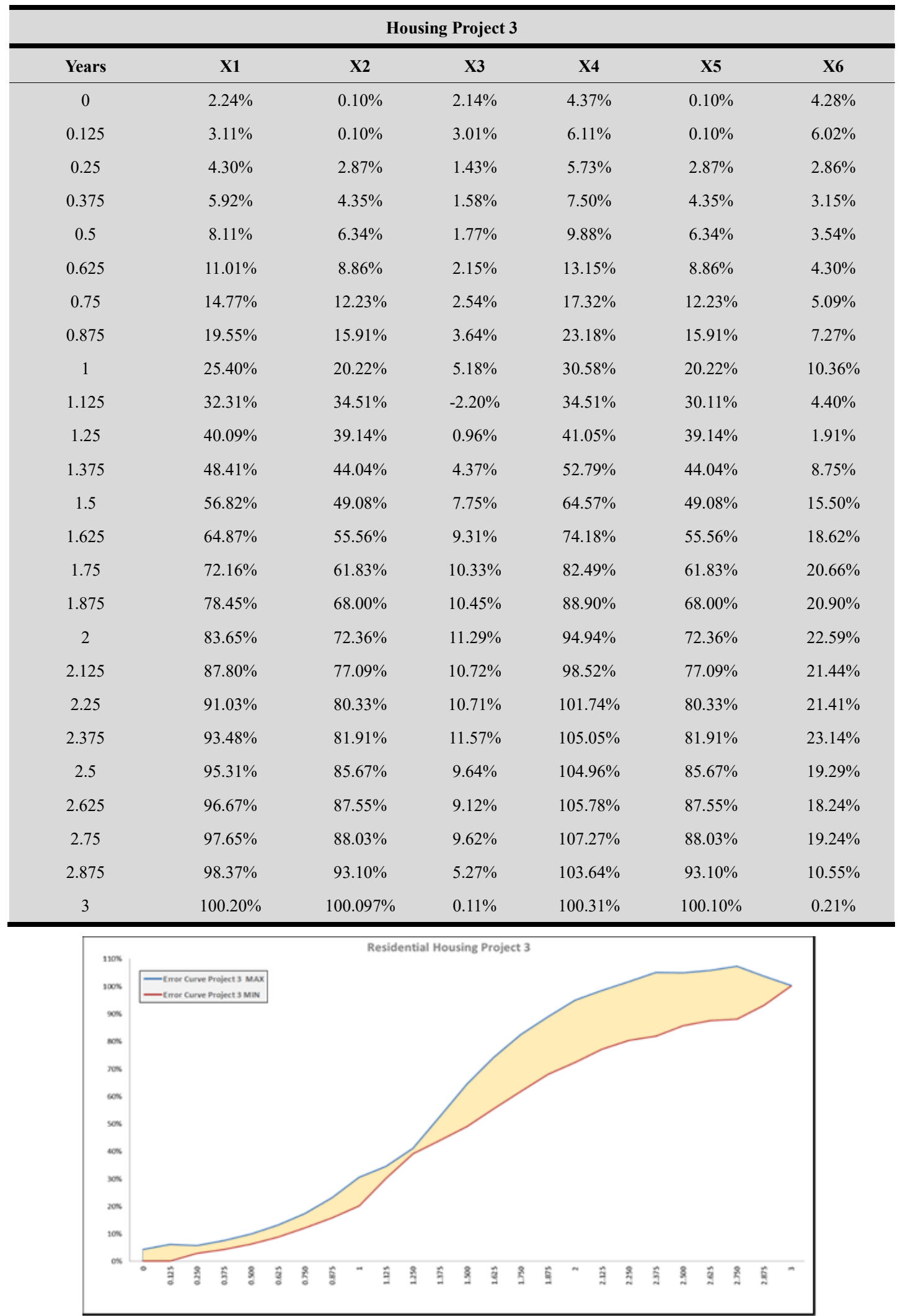

Figure 15. Cash flow area method range of verification project 3 (i.e., within the highlighted area). 
Journal of Entrepreneurship and Project Management

ISSN 2520-9116 (Online)

Vol. 6, Issue No.2, pp 1 -37, 2021

www.carijournals.org

Table 15. It is showing ALL verification projects and how much cost cash flow can fluctuate each time step.

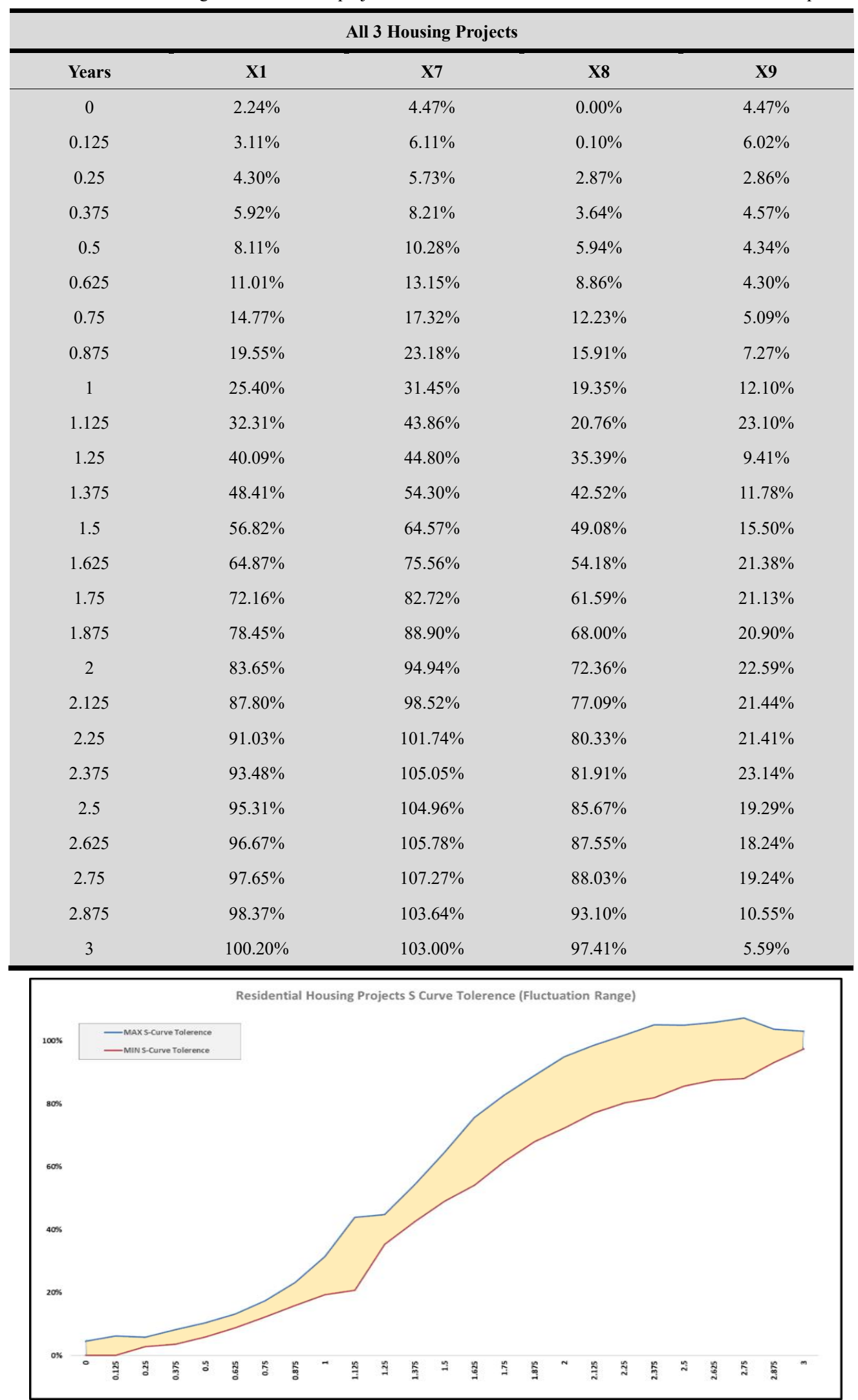

Figure 16. Cash flow area method range of ALL verification project $1,2 \& 3$ (i.e., within the highlighted area). 


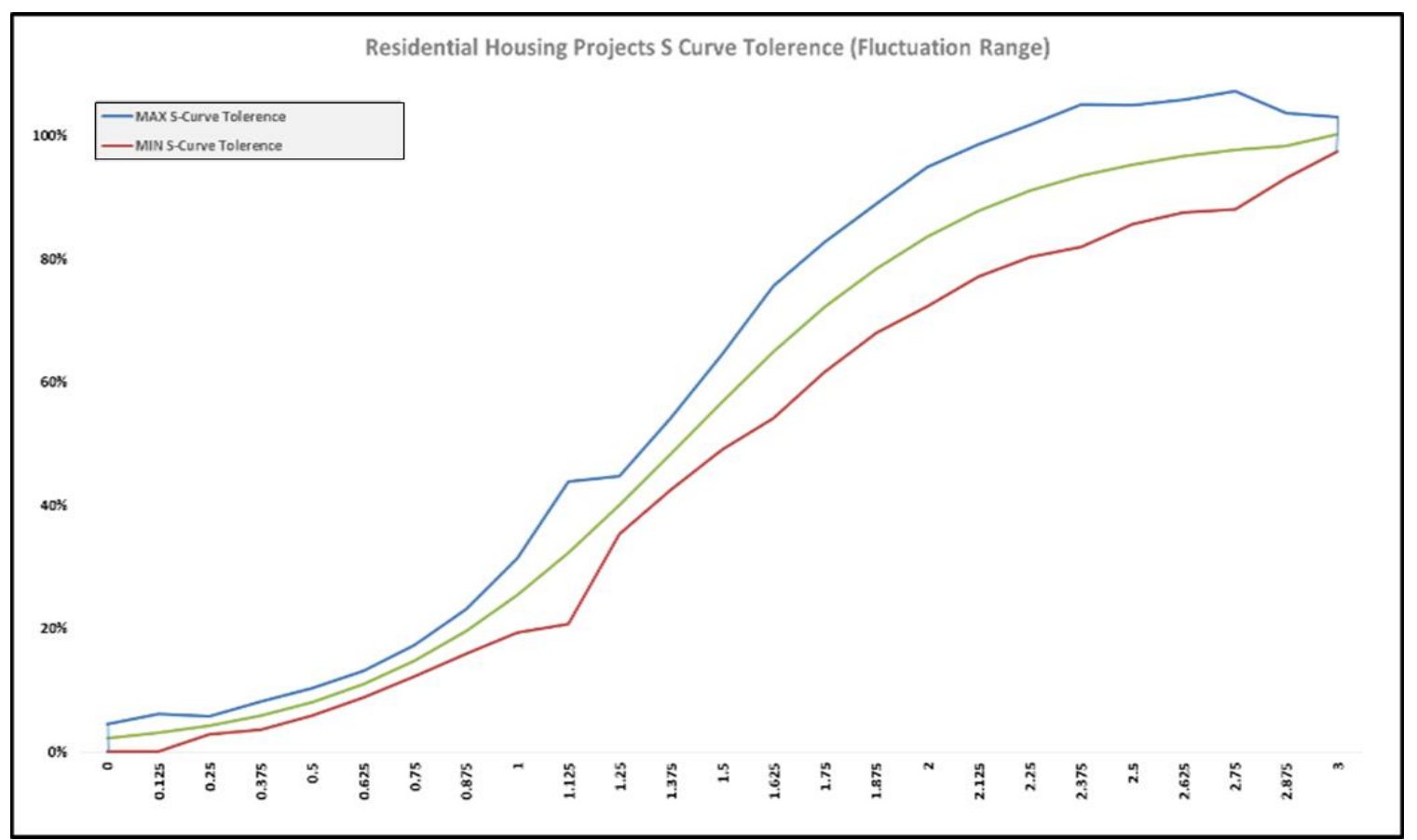

Figure 17. Cash flow area range of total verification projects 1, 2 and 3 including maximum limits, minimum limits, and estimated cash flow $S$-Curve.

However, this research is adding to the body of knowledge a new area method approach based on having a lower number than 30 projects. This research will include the maximum absolute value from actual and estimated curves at each time step. The highest limit contains all projects (i.e., three projects) and cash flow estimating curve shown in Figures 14, 15, 16, and 17. However, the final total tolerance range for better housing projects cash flow planning is shown in Figure 8. Nevertheless, those curves are based on three chosen projects only, and it is recommended to increase it to at least 30 (Konior \& Szóstak 2020). Then, validate it using SPSS to exclude the outlier projects (Konior \& Szóstak 2020). As more projects enter the process, the created area range in Figures 17 and 18 will be more accurate and include all similar projects' cash flow possibilities as shown in figure 8 which is done in previous research for hotel projects (Konior \& Szóstak 2020). 
Journal of Entrepreneurship and Project Management

ISSN 2520-9116 (Online)

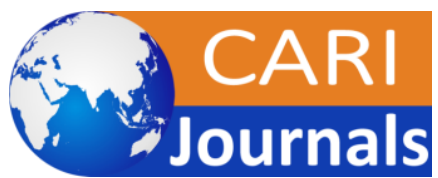

Vol. 6, Issue No.2, pp 1 -37, 2021

www.carijournals.org

\section{Construction Projects' Cash Flow Area Method Approach}

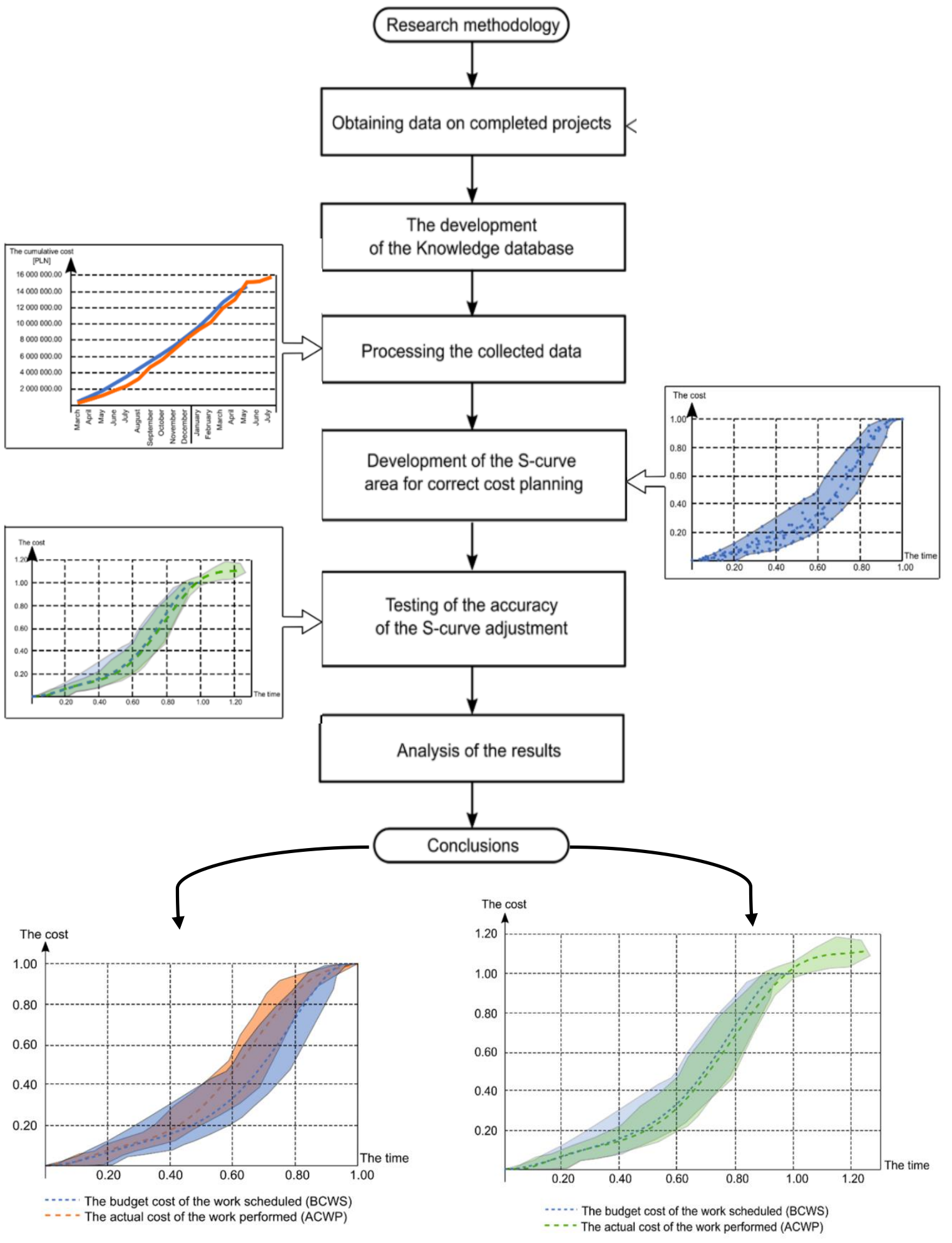

Figure 18: Construction projects' cash flow area method approach for accurate estimation in each time step over project's duration for hotel projects: an example for the existing previous area method approach (i.e., at least 30 projects) (Konior \& Szóstak 2020). 


\section{FINDINGS}

To sum up, this article introduced cash flow and risk s-curves establishment from client perspective using contracts tender value at the preconstruction stage. The mathematical calculations and figures explained how each step of the development was approached and completed. However, the originality, implications, and limitations will be presented as the following.

\section{CONCLUSION AND RECOMMENDATION}

To sum up, this article introduced cash flow and risk s-curves establishment from client perspective using contracts tender value at the preconstruction stage. The mathematical calculations and figures explained how each step of the development was approached and completed. However, the originality, implications, and limitations will be presented as the following.

\subsection{Originality}

According to the best knowledge of the author, there is no previous research approached standardizing cash flow and risk s-curves using simple logistic curve from client perspective at the preconstruction stage. Also, no previous research included the new approach of creating cash flow estimation area.

\subsection{Theoretical Implications}

This article is providing three main theoretical implications. It is presenting a new simple approach to create (1) construction cash flow increasing s-curve and (2) construction risk decreasing s-curve. Then, this research is introducing a new simple approach to establish construction project's cash flow stochastic values range (i.e., area method). The risk decreasing s-curve is considered the risk behaviour during construction; however, future research can use this behaviour to measure risk impact during construction stage.

\subsection{Practical Implications}

This article provides two important practical implications. First, the estimation cash flow s-curve is developed mathematically using the contract tender value solely. This will facilitate to experts to evaluate construction project's budget and actual-cost s-curves. The developed cost-percentage s-curve is based on zero-risk impact and is from client perspective at the preconstruction stage; however, experts in the professional industry will have the ability to adapt risks cost for better cash flow evaluation. The risk impact will be based on multiplying the collected risks probabilities by the risk behaviour s-curve. Then, multiply the risk impact percentage by the spent cash quantity in each time step (i.e., spent cash = cash flow s-curve multiplied by the contract tender value at the preconstruction stage). Finally, add the risk impact to the estimated zero-risk cash flow s-curve in terms of money. Nevertheless, the developed area method can adapt as many historical projects as possible to increase the estimation accuracy and minimize potential errors. The provided methods can be adapted to fit deferent construction lifetime (i.e., equal to 3 years in this article). 


\subsection{Limitations and Future Research Recommendations}

The limitations of this study are summarized as the following. Cash flow increasing S-Curve in this research, is analysed using three projects real housing projects. This is by identifying the yield absolute maximum points upper or lower the middle risk S-Curve. It will create a stochastic approach by developing a mirror area around the model's mean S-Curve values. Moreover, each time step will possibly move up and down within the identified area limits. However, this research agrees with previous researchers to increase the number of projects, in future research, to be more than 30 and establish a more accurate stochastic cash flow estimation.

\section{REFERENCES}

[1] Abanda, F. H., Tah, J. H. M. and Cheung, F. K. T. (2013). Mathematical modelling of embodied energy, greenhouse gases, waste, timecost parameters of building projects: A review, Building and Environment. vol. 59(1), pp. 23-37.

[2] Acebes, F., Pajares, J., Galán, J. M. and López-Paredes, A. (2013). Beyond Earned Value Management: A Graphical Framework for Integrated Cost, Schedule and Risk Monitoring', Procedia - Social and Behavioral Sciences, vol. 74(1), pp. 181-189.

[3] Adey, B. T., Herrmann, T., Tsafatinos, K., Lüking, J., Schindele, N. and Hajdin, R. (2012). Methodology and base cost models to determine the total benefits of preservation interventions on road sections in Switzerland, Structure and Infrastructure Engineering, vol. 8(7), pp. 639-654.

[4] Ahiaga-Dagbui, D. D. and Smith, S. D. (2014). Dealing with construction cost overruns using data mining, Construction Management and Economics, vol. 32 (7-8), pp. 682-694.

[5] Akinyemi, B., Ojiako, U., Maguire, S., Steel, G. and Anyaegbunam, A. (2009). Nigerian Banks and the Perception of Risk in PPP Project Delivery, Journal of Finance and Management, vol. 8(2), pp. 1-20.

[6] Aldwaik, M. and Adeli, H. (2016). Cost optimization of reinforced concrete flat slabs of arbitrary configuration in irregular high rise building structures, Structural and Multidisciplinary Optimization, vol. 54(1), pp. 151-164.

[7] Ali, A. S. and Kamaruzzaman, S. N. (2010). Cost Performance for Building Construction Projects in Klang Valley, Journal of Building Performance, vol. 1(1), pp. 110-118.

[8] An, S. H., Kim, G. H. and Kang, K. I. (2007). A case-based reasoning cost estimating model using experience by analytic hierarchy process, Building and Environment, vol. 42(7), pp. 2573-2579.

[9] Ayangade, J. A., Wahab, A. B. and Alake, O. (2009). An Investigation of the Performance of Due Process Mechanism in the Execution of Construction Projects in Nigeria, Civil Engineering Dimension, vol. 11(1), pp. 1-7.

[10] Baloi, D. and Price, A. D. F. (2003). Modelling global risk factors affecting construction cost performance, International Journal of Project Management, vol. 21(4), pp. 261-269.

[11] Battistoni, G., Genco, M., Marsilio, M., Pancotti, C., Rossi, S. and Vignetti, S. (2016). Cost-benefit analysis of applied research infrastructure. Evidence from health care, Technological Forecasting and Social Change, vol. 112(1), pp. 79-91.

[12] Cohen, M. A. and Kunreuther, H. (2007). Operations risk management: Overview of Paul Kleindorfer's contributions, Production and Operations Management, vol. 16(5), pp. 525-541.

[13] Cristóbal, J. R. S. (2017). The S-curve envelope as a tool for monitoring and control of projects', Procedia Computer Science, vol. 121(1), pp. 756-761.

[14] Dowd, K. and Cotter, J. (2007). Exponential Spectral Risk Measures, Journal of Economic Literature, vol. $15(1)$, pp. 1-16.

[15] Dikmen, I., Birgonul, M. T. and Han, S. (2007). Using fuzzy risk assessment to rate cost overrun risk in international construction projects, International Journal of Project Management, vol. 25(5), pp. 494-505.

[16] Doloi, H. (2012). Understanding impacts of time and cost related construction risks on operational performance of PPP projects, International Journal of Strategic Property Management, vol. 16(3), pp. 316-337.

[17] Eden, C., Williams, T. and Ackermann, F. (2005). Analysing project cost overruns: Comparing the "measured mile"; analysis and system dynamics modelling, International Journal of Project Management, vol. 23(2), pp. 135-139. 
[18] Hamaker, J. W. and Componation, P. J. (2005). Improving Space Project Cost Estimating With Engineering Management Variables, Engineering Management Journal, vol. 17(2), pp. 28-33.

[19] Hernandez-Sancho, F., Molinos-Senante, M. and Sala-Garrido, R. (2010). Cost modelling for wastewater treatment processes, Desalination, vol. 268(1), pp. 1-5.

[20] Howell, G. and Koskela, L. (2000). Reforming project management: the role of lean construction, Proceedings of the 8th Annual Conference of the International Group for Lean Construction. Brighton, UK, 17-19 Jul 2000.

[21] $\mathrm{Hu}, \mathrm{W}$. and He, X. (2014). An innovative time-Cost-Quality tradeoff modeling of building construction project based on resource allocation, The Scientific World Journal. Hindawi Publishing Corporation, vol. 1(1), pp. 1-10.

[22] Isaksson, T (2002). MODEL FOR ESTIMATION OF TIME AND COST BASED ON RISK EVALUATION APPLIED ON TUNNEL PROJECTS. PhD Thesis. Stockholm, Sweden: Royal Institute of Technology.

[23] Jrade, A. and Lessard, J. (2015). An Integrated BIM System to Track the Time and Cost of Construction Projects : A Case Study, Journal of Construction Engineering, vol. 1(1), pp. 1-11.

[24] Konior, J. and Szóstak, M. (2020). Methodology of planning the course of the cumulative cost curve in construction projects, Sustainability, vol. 12(6), 1-22.

[25] Kucharavy, D. and De Guio, R. (2015). Application of logistic growth curve, Procedia Engineering, vol. 131(1), pp. $280-290$.

[26] Koo, C., Hong, T., Hyun, C. and Koo, K. (2010). A CBR-based hybrid model for predicting a construction duration and cost based on project characteristics in multi-family housing projects, Canadian Journal of Civil Engineering, vol. 37(5), pp. 739-752.

[27] Lee, J. Y., Lee, S., Woo, S. and Shin, D. H. (2011). Framework of the approximate cost estimating model for river dredging construction, KSCE Journal of Civil Engineering, vol. 15(1), pp. 33-42.

[28] Liu, Z., Xu, J., Li, Y., Wang, X. and Wu, J. (2012). Using system dynamics to study the logistics outsourcing cost of risk, Kybernetes, vol. 41(9), pp. 1200-1208.

[29] Maiwenn J.Al (2013). Cost-effectiveness acceptability curves revisited, PharmacoEconomics, vol. 31(2), pp. 93-100.

[30] Mättö, T. and Sippola, K. (2016). Cost Management in the Public Sector: Legitimation Behaviour and Relevant Decision Making, Financial Accountability \& Management, vol. 32(2), pp. 179-201.

[31] Memon, A. H., Rahman, I. A., Abdullah, M. R. and Asmi, A. (2010). Factors Affecting Construction Cost in Mara Large Construction Project: Perspective of Project Management Consultant, International Journal of Sustainable Construction Engineering \& Technology, vol. 1(2), pp. 41-54.

[32] Meyer, A.-M., Wheeler, S. B., Weinberger, M., Chen, R. C. and Carpenter, W. R. (2013). An Overview of Methods for Comparative Effectiveness Research, Seminars in Radiation Oncology, vol. 24(1), pp. 5-13.

[33] Mizell, C. and Malone, L. (2007). A Project Management Approach to Using Simulation for Cost Estimation on Large, Complex Software Development Projects, Engineering Management Journal, vol. 19(4), pp. 28-34.

[34] Nasirzadeh, F., Khanzadi, M. and Rezaie, M. (2014). Dynamic modeling of the quantitative risk allocation in construction projects, International Journal of Project Management, vol. 32(3), pp. 442-451.

[35] Odeyinka, H. a., Lowe, J. and Kaka, A. P. (2013). Artificial neural network cost flow risk assessment model, Construction Management and Economics, vol. 31(5), pp. 423-439.

[36] Ong, A. S. L. and Ong, H. L. (1986). Modelling of Conveyance Construction Cost by Decomposition Technique, The Journal of the Operational Research Society, Vol. 37(10), pp. 979-985.

[37] Pajares, J. and López-Paredes, A. (2011). An extension of the EVM analysis for project monitoring: The Cost Control Index and the Schedule Control Index, International Journal of Project Management, vol. 29(5), pp. 615-621.

[38] Peng, D. X. and Lai, F. (2012). Using partial least squares in operations management research: A practical guideline and summary of past research, Journal of Operations Management, vol. 30(6), pp. 467-480.

[39] Petroutsatou, K. and Lambropoulos, S. (2010). Road tunnels construction cost estimation: A structural equation model development and comparison, Operational Research, vol. 10(2), pp. 163-173.

[40] Poh, Y. P. and Tah, J. H. M. (2006). Integrated duration-cost influence network for modelling risk impacts on construction tasks, Construction Management and Economics, vol. 24(8), pp. 861-868.

[41] Royal Institution of Chartered Surveyors (RICS), 2012. Retention 1st edition, guidance note. RICS QS \& Construction Standards GN 
90/2012. [online] Coventry: Royal Institution of Chartered Surveyors (RICS), pp.1-19.

[42] Schieg, M. (2010). Risk management in construction project management, Journal of Business Economics and Management, vol. 2(2), pp. 77-83.

[43] Seshadri, S. and Subrahmanyam, M. (2005). Introduction to the special issue on optimizing risk management in services, Production and Operations Management, vol. 14(1), pp. 1-4.

[44] Shah, R. and Goldstein, S. M. (2006). Use of structural equation modeling in operations management research: Looking back and forward, Journal of Operations Management, vol. 24(2), pp. 148-169.

[45] Sher, D. J. and Punglia, R. S. (2014). Decision analysis and cost-effectiveness analysis for comparative effectiveness research-a primer, Seminars in Radiation Oncology, vol. 24(1), pp. 14-24.

[46] Signor, R., Love, P. E. D., Olatunji, O., Marchiori, F. F. and Gripp, W. G. (2016). A Probabilistic Method for Forensic Cost Estimating of Infrastructure Projects, Applied Mathematical Modelling, vol. 21-22(1), pp. 1-15.

[47] Techet, A. H. (2005). Overview of basic probability, Design Principles for Ocean Vehicles, vol. 3(1), pp. 1-13.

[48] Thijssen, J. J. J. (2015). A model for irreversible investment with construction and revenue uncertainty, Journal of Economic Dynamics and Control, vol. 57, pp. 250-266.

[49] Toh, T.-C., Ting, C., Ali, K.-N., Aliagha, G.-U. and Munir, O. (2012). Critical Cost Factors of Building Construction Projects in Malaysia, Procedia - Social and Behavioral Sciences, vol. 57(1), pp. 360-367.

[50] Torp, O., Belay, A. M., Thodesen, C. and Klakegg, O. J. (2016). Cost Development Over-time at Construction Planning Phase: Empirical Evidence from Norwegian Construction Projects, Procedia Engineering, vol. 145(1877), pp. 1177-1184.

[51] Wang, F., Ding, L., Love, P. E. D. and Edwards, D. J. (2016). Modeling tunnel construction risk dynamics: Addressing the production versus protection problem, Safety Science, vol. 87(1), pp. 101-115.

[52] Wang, K., Wang, W., Wang, H., Hsu, P., Wu, W. and Kung, C. (2016). Automation in Construction Applying building information modeling to integrate schedule and cost for establishing construction progress curves, Automation in Construction, vol. 72(1), pp. 397410.

[53] Wilke, T. J. (2005). The Project Cost Variance Analysis Model: A Project Management Tool, The Journal of Cost Analysis \& Management, vol. 1656(2), pp. 13-23.

[54] Xu, J. W. and Moon, S. (2013). Stochastic Forecast of Construction Cost Index Using a Cointegrated Vector Autoregression Model, Journal of Management in Engineering, vol. 29(1), pp. 10-18.

[55] Zakis, K., Zakis, V. and Arfridsson, J. (2017). Eleven Nearly Zero New Building Life Cycle Cost and Dynamic Performance Optimization by Computer Modeling in Cold Climate, Procedia Computer Science, vol. 104(December), pp. 302-312.

[56] Zeng, J., An, M. and Smith, N. J. (2007). Application of a fuzzy based decision making methodology to construction project risk assessment, International Journal of Project Management, vol. 25(6), pp. 589-600.

[57] Zhu, X., Ruiz, R., Li, S. and Li, X. (2017). An effective heuristic for project scheduling with resource availability cost, European Journal of Operational Research, vol. 257(3), pp. 746-762. 